Ilchmann, Achim :

Non-identifier-based adaptive control of dynamical systems: a survey

Zuerst erschienen in:

IMA Journal of Mathematical Control and Information 8 (1991), S. 321-366 


\title{
Non-Identifier-Based Adaptive Control of Dynamical Systems: A Survey
}

\author{
ACHIM ILCHMANN \\ University of Exeter, Centre for Systems and Control Engineering, \\ School of Engineering, North Park Road, \\ Exeter, Devon EX4 $4 Q F$, U.K. \\ ilchmann.a@exeter.ac.uk
}

[Received 28 August 1991 and in revised form 12 November 1991]

\begin{abstract}
In adaptive control, the objective is to provide a single controller (consisting of a feedback law and a parameter adaptation law) which can control each system belonging to a certain class of systems. The systems are not known precisely: only structural properties (e.g. minimality, minimum phase, known relative degree) are assumed to hold. The control objectives are stabilization, tracking or servomechanism action. The paper surveys those aspects of the field of adaptive control which started in the 1970s wherein no parameter estimators are used. In addition to universal adaptive controllers for finite dimensional minimum phase systems of relative degree 1 , controllers for higher relative degree, non-minimum phase, infinite-dimensional, and nonlinear systems are also presented.
\end{abstract}

\section{Introduction}

A WIDE range of control theory deals with the problem that, for a known plant, a controller has to be designed in order that the feedback system achieves a prespecified control objective. The fundamental difference between this approach and that of adaptive control is that the plant is not known exactly, only structural information is available. The aim is therefore to design a single controller which can be applied to a variety of systems belonging to a certain class. The control law has to be designed so that the controller learns from the behaviour of the system, and based on this information, it adjusts its parameters. This area has been intensively studied over the last 40 years. See Åström (1987) for a survey article.

Up to the end of the $1970 \mathrm{~s}$, most adaptive control mechanisms would attempt to identify or to estimate certain parameters of the plant, and then design a feedback controller on the basis of this information. In this survey, an overview is given on adaptive controllers which are not based on any parameter identification or estimation algorithm or injections of probing signals. The objective is not to obtain information about the plant, but simply to control the unknown plant or process. For a conceptual framework, containing the controllers described in the present paper and, in addition, adaptive control systems formulated in terms of error models 
based on identification mechanisms, see Morse (1990), (1990a).

Most of the adaptive controllers surveyed in the present paper fit into the following general description. Suppose $\Sigma$ is a certain class of linear finite dimensional time-invariant systems of the form

$$
\left.\begin{array}{l}
\dot{x}(t)=A x(t)+B u(t)+E_{1} w, \quad y(t)=C x(t)+E_{2} w, \quad x(0) \in \mathbb{R}^{n} \\
\left(A, B, C, E_{1}, E_{2}\right) \in \mathbb{R}^{n \times n} \times \mathbb{R}^{n \times m} \times \mathbb{R}^{p \times m} \times \mathbb{R}^{n \times n_{1}} \times \mathbb{R}^{p \times n_{1}},
\end{array}\right\}
$$

$n_{1}, m, p$ are usually fixed, but $n$ is an arbitrary and unknown number, $y_{r e f}$ belonging to a (known) class of reference signals $\mathcal{Y}_{\text {ref }}$, and $w$ belongs to a (known) class of disturbance signals $\mathcal{D}$. It is desired to design a feedback law

$$
u(t)=f\left(k(t), y(t), y_{r e f}(t)\right)
$$

depending on the reference signal, the system output, and a 'tuning' parameter $k$ generated by

$$
\dot{k}(t)=g\left(k(t), y(t), y_{r e f}(t)\right), \quad k(0) \in \mathbb{R}^{l}
$$

so that there exists a (unique) solution of the closed loop system (1.1)-(1.3) on $[0, \infty)$, the internal variables are bounded, and most importantly $y(t)$ asymptotically tracks $y_{\text {ref }}(t)$.

Definition 1.1. Let $f: \mathbb{R}^{l} \times \mathbb{R}^{p} \times \mathbb{R}^{p} \rightarrow \mathbb{R}^{m}, \quad g: \mathbb{R}^{l} \times \mathbb{R}^{p} \times \mathbb{R}^{p} \rightarrow \mathbb{R}^{l}$ be continuous in $y$ and $y_{r e f}$, and piecewise right continuous in $k$. The controller consisting of the feedback law (1.2) and the adaptation law (1.3) is called a universal adaptive regulator solving the servomechanism problem for the class of systems $\Sigma$, the class of disturbances $\mathcal{D}$ and of reference signals $\mathcal{Y}_{\text {ref }}$, if for every $w \in \mathcal{D}, y_{\text {ref }} \in \mathcal{Y}_{\text {ref }}$, and every system (1.1) belonging to $\Sigma$ the closed system (1.1)-(1.3) satisfies

(i) there exists a (unique) solution on $\mathbb{R}_{+}$

(ii) $x, y, u$ are bounded if $y_{r e f}$ and $w$ are bounded

(iii) $\lim _{t \rightarrow \infty}\left[y(t)-y_{r \in f}(t)\right]=0$

(iv) $\lim _{t \rightarrow \infty} k(t)=k_{\infty} \in \mathbb{R}^{l}$ exists.

$l$ is called the order of the controller.

A (universal) adaptive regulator is called a (universal) adaptive tracking controller if $\mathcal{D}=\{0\}$, and a (universal) adaptive stabilizer if $\mathcal{D}=\{0\}$ and $\mathcal{Y}_{\text {ref }}=\{0\}$.

If the closed-loop system does not have the property of uniqueness of solutions, then (ii) and (iii) must be valid for every solution.

We also introduce adaptive controllers which do not fit into the descriptions given in Definition 1.1, i.e. $k(\cdot)$ in (1.3) not being generated by a differential equation, $f$ and $g$ depend on time, or $\Sigma$ is a class of infinite-dimensional or nonlinear systems. However, an extension of the above definitions to these cases is straightforward. The model reference problem is also covered by the tracking problem since the class of reference signals can be identified with a class of reference models and its inputs. 
The knowledge of the system, disturbance and reference classes is crucial for the design of simple adaptive controllers. If the system class consists of single-input single-output systems of the form

$$
\left.\begin{array}{l}
\dot{x}(t)=A x(t)+b u(t), \quad y(t)=c x(t), \quad x(0) \in \mathbb{R}^{n} \\
(A, b, c) \in \mathbb{R}^{n \times n} \times \mathbb{R}^{n} \times \mathbb{R}^{1 \times n}
\end{array}\right\}
$$

and the problem of adaptive stabilization is studied then the following assumptions are known as standard assumptions

(A1) The sign of the high frequency gain is known.

(A2) An upper bound on the order $n$ of the process is known.

(A3) The relative degree of the plant is known.

(A4) The system $(A, b, c)$ is minimum phase (see Definition 3.1).

Over the last 15 years, various authors have investigated the necessity of these conditions, how they can be generalized for larger classes, if they can be relaxed and how to design simple universal adaptive controllers. A chronological list of the most important contributions is as follows.

The first adaptive stabilizer, not based on identification of the system parameter and being universal for the class of single-input single-output systems satisfying only the assumptions (A1)-(A4), was given by Feuer and Morse (1978). This approach was improved in the following years; however, the controllers use full state observers and are thus complicated in nature. The first very simple controller exploring the high-gain properties of minimum phase systems was introduced by Willems and Byrnes (1984). They showed that the controller $\dot{k}=y^{2}, u=-\operatorname{sgn}(c b) k y$ is a universal adaptive stabilizer for all systems of the form (1.4) satisfying (A1), (A4), and having relative degree 1 . The open question, see Morse (1983), as to whether the knowledge of the sign of the high frequency gain (A1) is a necessary condition for adaptive stabilization was answered by Nussbaum (1983), who presented an adaptive stabilizer for first order single-input single-output systems where the sign of $c b$ is unknown. A very early contribution was made independently by Mareels (1984) who introduced a simple controller for the class (1.4) satisfying (A1), (A3), (A4), he used the 'internal model principle' to solve the tracking problem. It was shown by Mårtensson (1985), (1986) that the assumptions (A1)-(A4) can be weakened considerably. He proved the general result that the order of any stabilizer is a sufficient information for adaptive stabilization, Byrnes et al. (1986) showed the 'almost' converse. These results opened up a whole area of research devoted to the problems of non-identifier-based adaptive control. It was shown that the Willems-Byrnes controller can be used for multivariable minimum phase systems, being robust with respect to certain input and output nonlinearities, and also the tracking problem was solved. Extensions to nonlinear and to infinite-dimensional systems were given. 'The controller suggested by Mårtensson (1985),(1986) was simplified by Miller and Davison (1988) and used to solve the servomechanism problem. The idea of using discontinuous feedback has been introduced by Ryan (1988) to 
solve the stabilization and tracking problem for much larger classes of systems including certain nonlinear systems. Miller and Davison (1991) presented a controller which solves a modified tracking problem for minimum phase systems, exhibiting a
much better transient behaviour.

The aim of the research can be divided into two groups. One aim is to give necessary and sufficient conditions for classes of systems to solve the stabilization, tracking or servomechanism problem. These results are existential in nature: they show feasibility. The other aim is to design simple adaptive controllers which can easily be implemented. Mathematically, the controllers can be distinguished by whether or not they depend continuously on $k$. If a switching gain type controller is considered then the feedback gain switches at discrete points of time and between these points $k$ is held constant. Therefore the resulting closed loop system is a piecewise time-invariant linear system coupled with a scalar nonlinear equation. This simplifies the analysis.

The paper is organized in a systematical rather than in a chronological way. In order to make the reader familiar with some basic techniques used in this area, some proofs are given explicitly. We start in Section 2 with the most elementary class of systems, namely first order systems. Even in this simple situation we gain insight into basic ideas as high-gain, switching strategy, and necessary and sufficient conditions. In Section 3 , the class of multivariable linear systems which are minimum phase and of relative degree 1 is studied. After presenting basic properties, we introduce various controllers and extend the system class step by step by generalizing the assumption on the high frequency gain. In Section 4 , the assumption of relative degree 1 is dropped. Eventually, non-minimum phase systems are studied in Section 5. Principal results on adaptive stabilization and model reference control are reported in Section 6. In Section 7 (respectively, 8) it is shown how previous results could be extended in an infinite-dimensional (respectively, nonlinear) set-up. Open problems and future research is discussed in Section 9.

\section{Contents}

2. Single input single output systems of order 1 .

3. Multivariable minimum phase systems of relative degree 1.

3.1 Properties of minimum phase systems

$3.2 \sigma(C B) \subset \mathbb{C}_{+}$

$3.3 \sigma(C B) \subset \mathbb{C}_{-} \quad$ or $\quad \sigma(C B) \subset \mathbb{C}_{+}$

$3.4 \operatorname{det}(C B) \neq 0$

3.5 Exponential stabilization

3.6 Tracking

3.7 Robustness

4. Minimum phase systems of higher relative degree.

4.1 Observer-based model reference controllers

4.2 Non-observer-based stabilization and tracking

4.3 Tracking within a ball

5. Non minimum phase systems.

5.1 Stabilization

5.2 Tracking and the servomechanism problem

6. Necessary and sufficient conditions. 
7. Infinite-dimensional systems.

8. Nonlinear systems.

9. Conclusions

\section{Single-input single-output systems of order 1}

In this section, we consider the simplest system class, namely single-input, single-output systems of order 1 . In this case we gain insight into the idea of highgain adaptive control, the concept of switching functions, and characterizations of universal adaptive stabilizers.

Consider the class of controllable and observable scalar systems of the form

$$
\left.\begin{array}{l}
\dot{x}(t)=a x(t)+b u(t), \quad y(t)=c x(t), \quad x(0) \in \mathbb{R} \\
a, b, c \in \mathbb{R} \text { and } \quad c b \neq 0
\end{array}\right\}
$$

If we apply the feedback law $u(t)=-k y(t)$ to (2.1) then the closed loop system has the form

$$
\dot{x}(t)=[a-k c b] x(t)
$$

Clearly, if $a /|c b|<|k|$ and $\operatorname{sign}(k)=\operatorname{sign}(c b)$, then $(2.2)$ is exponentially stable. However, $a, b, c$ are not known and so the problem is to find adaptively an appropriate $k$ so that the motion of the feedback system tends to zero.

Consider first the subclass where the high frequency gain is positive

$$
(a, b, c) \text { belongs to class }(2.1) \text { and satisfies } c b>0 \text {. }
$$

Now a time-varying feedback is build into the feedback law

$$
u(t)=-k(t) y(t)
$$

where $k(t)$ has to be adjusted so that it converges to a finite limit which is large enough to ensure stability. This can be achieved by the adaptation rule

$$
\dot{k}(t)=|y(t)|^{p}, \quad k(0) \in \mathbb{R}
$$

where $p \geq 1$ is arbitrary. The nonlinear closed-loop system

$$
\dot{x}(t)=[a-k(t) c b] x(t), \quad k(t)=|c|^{p} \int_{0}^{t}|x(s)|^{p} d s+k(0), \quad(k(0), x(0)) \in \mathbb{R} \times \mathbb{R}
$$

has at least a solution on a small interval $\left[0, t^{\prime}\right)$, and the solution

$$
x(t)=\exp \left\{\int_{0}^{t}[a-k(s) c b] d s\right\}+x(0)
$$

is exponentially increasing so long as $a-k(t) c b>0$. Hence, $k(t) \geq t|c x(0)|^{p}+k(0)$ also increases. Therefore, there exists a $t^{*} \geq 0$ such that $a-k\left(t^{*}\right) c b=0$ and (2.6) yields $a-k(t) c b<0$ for all $t>t^{*}$. This yields that the solution $x(t)$ decays exponentially and $\lim _{t \rightarrow \infty} k(t)=k_{\infty} \in \mathbb{R}$ exists. So we have proved that (2.4), $(2.5)$ is a universal adaptive stabilizer for the class (2.3). 
Furthermore, as S. Townley of the University of Exeter pointed out to the author, in this simple situation it is even possible to determine the terminal gain $k_{\infty}:=$ $\lim _{t \rightarrow \infty} k(t)$ in terms of the system data. If, for example, $p=2$ in $(2.5)$, then it follows from (2.2) that

$$
y(t) \dot{y}(t)=(a-k(t) c b) \dot{k}(t)
$$

and integration yields

$$
\frac{y(t)^{2}}{2}-\frac{y(0)^{2}}{2}=\int_{0}^{t}(a-k(s) c b) \dot{k}(s) d s=\int_{k(0)}^{k(t)}(a-\tau c b) d \tau
$$

Since $\lim _{t \rightarrow \infty} x(t)=0, k_{\infty}$ is the positive solution of the quadratic equation

$$
-\frac{y(0)^{2}}{2}=a\left[k_{\infty}-k(0)\right]-\frac{c b}{2}\left[k_{\infty}^{2}-k(0)^{2}\right]
$$

which is

$$
k_{\infty}=\frac{a}{c b}+\sqrt{\left(\frac{a}{c b}-k(0)\right)^{2}+\frac{y(0)^{2}}{2}} .
$$

It was an open problem as to whether there exists a universal adaptive stabilizer for the class (2.1). Morse (1983) conjectured that there does not exist a universal adaptive stabilizer if $f$ and $g$, see (1.2) and (1.3), are differentiable functions. This would imply that the knowledge of the high frequency gain is a necessary condition for universal adaptive stabilization, see (A1). Nussbaum (1983) proved that the conjecture is true if $f$ and $g$ are required to be polynomials or rational functions. More importantly, Nussbaum introduced the following rich class of analytic controllers which are universal adaptive stabilizers.

$$
u(t)=\left[k(t)^{2}+1\right] h(k(t)) y(t), \quad \dot{k}(t)=y(t)\left[k(t)^{2}+1\right], \quad k(0) \in \mathbb{R}
$$

where $h: \mathbb{R} \rightarrow \mathbb{R}$ is an analytic function satisfying

$$
\sup _{k>0} \frac{1}{k} \int_{0}^{k} h(s) d s=+\infty \text { and } \inf _{k>0} \frac{1}{k} \int_{0}^{k} h(s) d s=-\infty
$$

Nussbaum's example is

$$
h(k)=\cos \left(\frac{\pi}{2} k\right) \exp \left\{k^{2}\right\} .
$$

The intuition behind this controller is that the switching function $h(\cdot)$ takes both positive and negative values and the sign is kept constant on longer and longer intervals. Eventually $h(\cdot)$ has the 'correct' sign for a sufficiently long period in which the closed loop system stabilizes out such that $k(t)$ converges to a finite limit and no more switching occurs.

Willems and Byrnes (1984) proved that the following simplification of (2.7) is a universal adaptive stabilizer for the class (2.1) as well

$$
u(t)=N(k(t)) k(t) y(t), \quad \dot{k}(t)=y(t)^{2}, \quad k(0) \in \mathbb{R}
$$


where $N: \mathbb{R} \rightarrow \mathbb{R}$ is a Nussbaum function, i.e. a piecewise right-continuous function which satisfies the so called Nussbaum condition

$$
\sup _{k>0} \frac{1}{k} \int_{0}^{k} N(\tau) \tau d \tau=+\infty \quad \text { and } \quad \inf _{k>0} \frac{1}{k} \int_{0}^{k} N(\tau) \tau d \tau=-\infty
$$

Examples are given by $N_{1}(t)=\sin \sqrt{t}, N_{2}(t)=t \cos t$, and

$$
N_{3}(t)=\left\{\begin{array}{rlll}
1 & \text { if } & 0 \leq|t|<\lambda_{0} \\
1 & \text { if } & \lambda_{n} \leq|t|<\lambda_{n+1}, & n \text { even } \\
-1 & \text { if } & \lambda_{n} \leq|t|<\lambda_{n+1}, & n \text { odd }
\end{array}\right.
$$

where, for example, $\lambda_{n}:=n^{2}$ or $\lambda_{n+1}:=\lambda_{n}^{2}, \lambda_{0}>1$.

The following stabilizer suggested by Morse (1984)

$$
u(t)=k(t)^{2} \cos k(t) y(t), \quad \dot{k}(t)=y(t)^{2}, \quad k(0) \in \mathbb{R}
$$

is a special case of (2.9).

The class (2.1) has been extensively studied by Helmke and Prätzel- Wolters (1988), a shortened version can be found in Helmke and Prätzel-Wolters (1988a). They have tried to characterize the set of all universal adaptive stabilizers of order 1 under the constraint that the feedback and adaptation law

$$
u(t)=f(k(t), y(t)), \quad \dot{k}(t)=k(t)^{r} g(k(t), y(t)), \quad k(0) \in \mathbb{R}, r \geq 1
$$

are given by analytic functions $f$ and $g$.

As a necessary condition they proved the following proposition.

PRoposition 2.1. If (2.12) is a universal adaptive stabilizer for the class (2.1) then necessarily

$$
\begin{gathered}
g(k, y)=0 \Longrightarrow y=0 \\
\text { and for all }(k, y) \in \mathbb{R}^{2} \text { either } g(k, y) \geq 0 \text { or } g(k, y) \leq 0
\end{gathered}
$$

That this condition is almost sufficient is shown in the following theorem.

THEOREM 2.2. Suppose $f, g$ are analytic functions, $g$ satisfies the necessary conditions (2.13), (2.14) and, in addition,

$$
g(k, y) \geq m \quad \text { for all } \quad(k, y) \in \mathbb{R}^{2} \backslash \mathcal{K}
$$

for some compact set $\mathcal{K} \subset \mathbb{R}^{2}$ and some $m>0$. Suppose furthermore, that

$$
\hat{f}:=a+c b \frac{f(k, y)}{y}
$$

is such that $\hat{f} / g$ can be decomposed into $\hat{f}(k, y) / g(k, y)=\tilde{f}(y)+h(k, y)$ with

$$
|h(k, y)| \leq M \quad \text { for all }(k, y) \in \mathbb{R}^{2} \backslash \mathcal{K}, \text { for some } M>0
$$


and the following Nussbaum type conditions are satisfied

$$
\begin{array}{lll}
\sup _{k>0} \frac{1}{k} \int_{0}^{k} \tilde{f}(\tau) d \tau=+\infty & \text { and } & \sup _{k>0} \frac{1}{k} \int_{0}^{k} \tilde{f}(\tau) d \tau=+\infty \\
\inf _{k>0} \frac{1}{k} \int_{0}^{k} \tilde{f}(\tau) d \tau=-\infty & \text { and } \quad \inf _{k>0} \frac{1}{k} \int_{0}^{k} \tilde{f}(\tau) d \tau=-\infty
\end{array}
$$

Then (总.12) is a universal adaptive stabilizer for the class (D. 1 ).

Examples for these controllers are the Nussbaum controller (2.7), the WillemsByrnes controller (2.9), and the following Heymann-Lewis Meyer controller

$$
u(t)=\left[k(t)+\frac{1}{2} y(t)^{2}\right]^{2} \cos \left(k(t)+\frac{1}{2} y(t)^{2}\right) y(t), \quad \dot{k}(t)=y(t)^{2}, \quad k(0) \in \mathbb{R}
$$

Heymann et al. (1985) proved certain terminal behaviour of this controller depending on the initial conditions of the system. It is shown that the transient behaviour of (2.17) is better than that of the Willems Byrnes controller (2.9) or of the Morse controller (2.11) whose terminal behaviour is unpredictable and dependent, in an erratic way, on the initial data.

Another improvement of the local behaviour of the controller (2.9), in the case of known high frequency gain, is achieved by Cabrera and Furuta (1989) who modify the adaptation law in (2.9) to $\dot{k}=-\sigma k+y^{2}$ for some $\sigma>0$. Under certain assumptions on the system class the closed loop system is robust against bounded
disturbances.

\section{Multivariable minimum phase systems of relative degree 1 .}

In this section, we consider classes of $m$-input $m$-output systems of the form

$$
\dot{x}(t)=A x(t)+B u(t), \quad y(t)=C x(t), \quad x(0) \in \mathbb{R}^{n}
$$

where $(A, B, C) \in \mathbb{R}^{n \times n} \times \mathbb{R}^{n \times m} \times \mathbb{R}^{m \times n}$, and $n$ is unknown.

\subsection{Properties of minimum phase systems}

Before presenting various adaptive controllers, we study one of the basic assumptions common to many classes of higher order systems.

DEFINITION 3.1. A system of the form (3.1) is called minimum phase if it satisfies

$$
\operatorname{det}\left[\begin{array}{cc}
s I-A & B \\
C & 0
\end{array}\right] \neq 0 \text { for all } s \in \overline{\mathbb{C}}_{+} \text {. }
$$

The minimum phase property can be characterized as follows.

PRoposition 3.2. The system $(A, B, C)$ of the form (3.1) is minimum phase if
and only if it satisfies the conditions

(i) $r k\left[s I_{n}-A, B\right]=n \quad \forall s \in \overline{\mathbb{C}}_{+}$, i.e. $(A, B)$ is stabilizable by state feedback.

(ii) $r k\left[\begin{array}{l}s I_{n}-A \\ C\end{array}\right]=n \quad \forall s \in \overline{\mathbb{C}}_{+}$, i.e. $(\dot{A}, C)$ is detectable. 
(iii) $C\left(s I_{n}-A\right)^{-1} B \in \mathbb{R}(s)^{m \times m}$ has no zeros in $\overline{\mathbb{C}}_{+}$.

Proposition 3.2 shows that, for stabilizable and detectable single-input singleoutput systems, condition (3.2) is equivalent to the well-known definition via the transfer function.

A relationship between multivariable minimum phase systems and positive real systems has been investigated in Owens et al. (1987).

If $\operatorname{det}(C B) \neq 0$ then the state space can be decomposed into the direct sum $\mathbb{R}^{n}=i m B \oplus \operatorname{ker} C$ and this leads to the following convenient decomposition of the system (3.1).

Lemma 3.3. Suppose (3.1) satisfies $\operatorname{det}(C B) \neq 0$. If $V \in \mathbb{R}^{n \times(n-m)}$ denotes a basis matrix of ker $C$, then $U:=\left[B(C B)^{-1}, V\right]$ has the inverse

$$
U^{-1}=\left[\begin{array}{c}
C \\
T
\end{array}\right], \text { where } T=\left(V^{T} V\right)^{-1} V^{T}\left[I_{n}-B(C B)^{-1} C\right] .
$$

Hence the state space transformation

$$
\left(\begin{array}{c}
y \\
z
\end{array}\right)=U^{-1} x=\left(\begin{array}{c}
C x \\
T x
\end{array}\right)
$$

converts (3.1) into

$$
\left.\begin{array}{l}
\dot{y}(t)=A_{1} y(t)+A_{2} z(t)+C B u(t) \\
\dot{z}(t)=A_{3} y(t)+A_{4} z(t)
\end{array}\right\}
$$

where $A_{1} \in \mathbb{R}^{m \times m}, A_{2} \in \mathbb{R}^{m \times(n-m)}, A_{3} \in \mathbb{R}^{(n-m) \times m}, A_{4} \in \mathbb{R}^{(n-m) \times(n-m)}$. If (3.1) is minimum phase then $\sigma\left(A_{4}\right) \subset \mathbb{C}_{-}$.

Consider a minimum phase system of the form (3.1) satisfying $\operatorname{det}(C B) \neq 0$ Then it is possible to choose $h \in \mathbb{R}^{m \times m}$ such that $\sigma(C B K) \subset \mathbb{C}_{+}$. If the feedback law $u(t)=-k K y(t)$ is applied to (3.1) then for $k$ large enough, i.e. high gain, the closed-loop system is stable. 'This follows from

$$
\begin{aligned}
& \operatorname{det}\left[\begin{array}{cc}
\lambda I-A_{1}+k C B K & -A_{2} \\
-A_{3} & \lambda I-A_{4}
\end{array}\right] \\
& =\operatorname{det}\left(\lambda I-A_{1}+k C B K\right) \operatorname{det}\left[\left(\lambda I-A_{4}\right)-A_{3}\left(\lambda I-A_{1}+k C B K\right)^{-1} A_{2}\right] \text {. }
\end{aligned}
$$

Thus, in the limit we obtain

$$
\lim _{k \rightarrow \infty} \sigma(A-k B k C)=\lim _{k \rightarrow \infty} \sigma(-k C B K) \cup \sigma\left(A_{4}\right)
$$

In the adaptive situation $k$ will be time-varying. However the previous intuition for the stability of the system remains valid and we have the so called high gain lemma.

LEMMA 3.4. Suppose $k(\cdot):\left[0, t^{\prime}\right) \rightarrow \mathbb{R}, t^{\prime} \leq \infty$, is an unbounded nondecreasing piecewise continuous function and

(i) $D \in \mathbb{R}^{n \times n}$ has simple null structure, i.e. the zero eigenvalues are semisimple, 
(ii) $\sigma(D) \backslash\{0\} \subset \mathbb{C}_{+}$,

(iii) for some $\epsilon>0$ there exists $t^{*} \in\left[0, t^{\prime}\right)$ such that

$$
\sigma(A-k(t) D) \subset\left\{\lambda \in \mathbb{C}_{-} \mid \operatorname{Re} \lambda<-\epsilon\right\} \quad \text { for all } t \in\left[t^{*}, t^{\prime}\right) .
$$

Then the system $\dot{x}(t)=[A-k(t) D] x(t)$ is exponentially stable, i.e. there exist $M, \lambda>0$ such that

$$
\|x(t)\| \leq M e^{-\lambda\left(t-t_{0}\right)}\left\|x\left(t_{0}\right)\right\| \quad \text { for all } t \in\left[t_{0}, t^{\prime}\right), t_{0} \in\left[0, t^{\prime}\right) .
$$

Lemma 3.4 has been stated independently by Mårtensson $(1986,1987)$ and by Ilchmann et al. (1987). However both proofs are incomplete. Schmid (1991) pointed out that, without assumption (ii), the claim of the lemma does not hold true in general. The proof in Ilchmann et al. (1987) goes through if (ii) is added.

If the system (3.1) fulfils $\sigma(C B) \subset \mathbb{C}_{+}$, then $D=B C$ obviously satisfies (i) and (ii), and the minimum phase condition implies (iii).

Another important consequence of the minimum phase property is the following inequality which relates the input and the output of the system only.

Proposition 3.5. Suppose the system (3.1) is minimum phase and $\operatorname{det}(C B) \neq 0$. Let $0 \leq t_{0}<t^{\prime} \leq \infty$ and $u(\cdot):\left[t_{0}, t^{\prime}\right) \rightarrow \mathbb{R}^{m}$ be measurable and locally integrable, $P \in \mathbb{R}^{n \times n}$ be positive definite, and $p \geq 1$. Then there exists an $M>0$ such that for all $t \in\left[t_{0}, t^{\prime}\right)$

$$
\frac{1}{p}\|y(t)\|_{P}^{p} \leq M+M \int_{t_{0}}^{t}\|y(s)\|_{P}^{p} d s+\int_{t_{0}}^{t}\|y(s)\|_{P}^{p-1}\langle\beta(y(s)), P C B u(s)\rangle d s
$$

where $\beta: \mathbb{R}^{m} \rightarrow \mathbb{R}^{m}, y \mapsto \beta(y):= \begin{cases}\|y\|_{P}^{-1} y, & y \neq 0 \\ 0, & y=0\end{cases}$

A proof is given in Ilchmann and Owens (1991a) and in a more detailed version in Ilchmann and Logemann (1991). Lemma 3.4 and Proposition 3.5 are crucial for the generalization of the Willems-Byrnes controller (2.9) to multivariable minimum phase systems. This will be done in the following two subsections.

\section{2. $\sigma(C B) \subset \mathbb{C}_{+}$}

In this subsection, we consider the class of multivariable systems of the form (3.1) which have an invertible high frequency gain $C B$ with unmixed spectrum. Let

$$
\left.\begin{array}{l}
\dot{x}(t)=A x(t)+B u(t), \quad y(t)=C x(t), \quad x(0) \in \mathbb{R}^{n} \\
(A, B, C) \in \mathbb{R}^{n \times n} \times \mathbb{R}^{n \times m} \times \mathbb{R}^{m \times n}, \quad \sigma(C B) \subset \mathbb{C}_{+} \\
(A, B, C) \quad \text { is minimum phase, } n \text { arbitrary }
\end{array}\right\}
$$

THEOREM 3.6. Let $p \geq 1$. Then the controller

$$
u(t)=-k(t) y(t), \quad \dot{k}(t)=\|y(t)\|^{p}, \quad k(0) \in \mathbb{R}
$$


is a universal adaptive stabilizer for the class (3.5), i.e. the solution of the nonlinear closed-loop system

$$
\left.\begin{array}{ll}
\dot{x}(t)=[A-k(t) B C] x(t), & x(0) \in \mathbb{R}^{n} \\
\dot{k}(t)=\|y(t)\|^{p}, & k(0) \in \mathbb{R}
\end{array}\right\}
$$

exists on the whole of $\mathbb{R}_{+}$, and

$$
\lim _{t \rightarrow \infty} y(t)=0, \quad \lim _{t \rightarrow \infty} k(t) \in \mathbb{R} \quad \text { exists. }
$$

Proof. Since the right hand side of (3.7) is locally Lipschitz in $x$ and in $k$, there exists a maximal $t^{\prime}>0$ such that (3.7) has a unique solution on $\left[0, t^{\prime}\right)$. Suppose $k(\cdot) \notin L_{\infty}\left(0, t^{\prime}\right)$. Then Lemma 3.4 implies that $x(\cdot)$ satisfies

$$
\|x(t)\| \leq M e^{-\lambda t}\|x(0)\| \text { for all } t \in\left[0, t^{\prime}\right) .
$$

and the adaptation rule implies $k(\cdot) \in L_{\infty}\left(0, t^{\prime}\right)$. Therefore the solution of (3.7) does not have a finite escape time, i.e. $t^{\prime}=\infty$. Since $y(\cdot) \in L_{p}(0, \infty)$, it follows from the second equation in (3.3) that $z(\cdot) \in L_{p}(0, \infty)$, thus $(3.3)$ yields $\dot{z}(\cdot) \in L_{p}(0, \infty)$. Now $x(\cdot), \dot{x}(\cdot) \in L_{p}(0, \infty)$ gives $\lim _{t \rightarrow \infty} x(t)=0$. This completes the proof.

Using the basic ideas of the previous proof, it can be shown that the class of adaptation rules can be extended as follows.

THEOREM 3.7. Let $p \geq 1$ and consider

$$
u(t)=-k(t) y(t), \quad \dot{k}(t)=g(t, k(t), y(t)), \quad k(0)=k_{0} \in \mathbb{R}
$$

where $g: \mathbb{R}_{+} \times \mathbb{R}_{+} \times \mathbb{R}^{m} \rightarrow \mathbb{R}$ is a Carathéodory function, locally Lipschit $z$ in the second and third argument, and locally integrable in $t \in \mathbb{R}_{+}$. Suppose that the solution $k(\cdot)$ of the closed-loop system (3.1), (3.8) satisfies, on its maximal interval of existence $\left[0 . t^{\prime}\right)$, the following conditions

$$
\begin{aligned}
& k(t) \geq 0 \text { and non-decreasing in } t \\
& y(\cdot) \in L_{i}\left(0, t^{\prime}\right) \text { for all } i \in[p, \infty] \Rightarrow k(\cdot) \in L_{\infty}\left(0, t^{\prime}\right) \\
& k(\cdot) \in L_{\infty}\left(0, t^{\prime}\right) \Rightarrow y(\cdot) \in L_{p}\left(0, t^{\prime}\right)
\end{aligned}
$$

Then (3.8) is a universal adaptive controller for the class (3.5).

EXAMPLE 3.8 .

$$
\dot{k}(t)=\sum_{i=p}^{p^{\prime}}\|y(t)\|^{i} F(y(t)), \quad 1 \leq p \leq p^{\prime}<\infty, \quad k(0) \geq 0
$$

where $F: \mathbb{R} \rightarrow \mathbb{R}$ is a polynomial such that $F(\lambda) \geq F_{0}>0$ for all $\lambda \in \mathbb{R}$.

That the feedback law $u(t)=-k(t) y(t)$ together with an appropriate adaptation law leads to a universal adaptive stabilizer for the class (3.5) has been shown for 
- $m=1$ and $\dot{k}(t)=\|y(t)\|^{2}$ by Willems and Byrnes (1984)

$\cdots \quad m \geq 1$ and $\dot{k}(t)=\|y(t)\|^{2}$ by Byrnes and Willems (1984)

- $m \geq 1$ and $\dot{k}(t)=\alpha\|y(t)\|^{2}+\beta\|u(t)\|^{2}, \alpha>0, \beta \geq 0$ by Mårtensson (1986)

- $m \geq 1, k(t)$ satisfying (3.9)-(3.11) for $p=2$ by Owens et al. (1987).

Owens (1991) has proved that the Willems-Byrnes controller is also applicable to a certain class of singular systems.

\section{3. $\sigma(C B) \subset \mathbb{C}_{+}$or $\sigma(C B) \subset \mathbb{C}$}

If the sign of $c b$ is unknown or the spectrum of $C B$ is known to lie either in the open right or left half plane, then Nussbaum's idea of implementing a switching function (see Section 2) carries over to the $n$-th order case. Consider the following class of all minimum phase systems of the form (3.1) with unknown state dimension and $\sigma(C B) \subset \mathbb{C}_{+}$or $\sigma(C B) \subset \mathbb{C}_{-}$, i.e.

$$
\left.\begin{array}{l}
\dot{x}(t)=A x(l)+B u(t), \quad y(t)=C x(t), \quad x(0) \in \mathbb{R}^{n} \\
(A, B, C) \in \mathbb{R}^{n \times n} \times \mathbb{R}^{n \times m} \times \mathbb{R}^{m \times n}, \quad \sigma(C B) \subset \mathbb{C}_{+} \text {or } \sigma(C B) \subset \mathbb{C}_{-} \\
(A, B, C) \text { is minimum phase, } n \text { arbitrary }
\end{array}\right\}
$$

Due to the multivariable situation, we need to introduce scaling invariant Nussbaum functions, i.e. piecewise right-continuous functions $N(\cdot): \mathbb{R} \rightarrow \mathbb{R}$ so that for every
$\alpha, \beta>0$, the function

$$
\tilde{N}(t)= \begin{cases}\alpha N(t) & \text { if } \quad N(t) \geq 0 \\ \beta N(t) & \text { if } \quad N(t)<0\end{cases}
$$

satisfies (2.10) as well. (The concept of scaling invariant switching functions was originally introduced by Logemann and Owens (1988).) THEOREM 3.9. Let $p \geq 1$ and $N: \mathbb{R} \rightarrow \mathbb{R}$ be a scaling invariant Nussbaum function.
Then the controller

$$
u(t)=-N(k(t)) k(t) y(t), \quad \dot{k}(t)=\|y(t)\|^{p}, \quad k(0) \in \mathbb{R}
$$

is a universal adaptive controller for the class (3.12).

Proof. The proof is similar to that of Theorem 3.6 , only the step that $k(\cdot) \notin$ $L_{\infty}\left(0, t^{\prime}\right)$ needs a modification. Without loss of generality, assume that $\sigma(C B) \subset \mathbb{C}_{+}$ (otherwise consider $-C B$ ). Let $P \in \mathbb{R}^{m \times m}$ be positive definite and such that $P C B+(C B)^{T} P=I_{m}$. Inserting the feedback law into the inequality (3.4) yields, for some $M>0$ and $k\left(t_{0}\right) \neq 0$

$$
\begin{aligned}
\frac{1}{p}\|y(t)\|_{P}^{p} & \leq M+M \int_{t_{0}}^{t}\|y(s)\|_{P}^{p} d s-\int_{t_{0}}^{t} N(k(s)) k(s)\|y(s)\|_{P}^{p-1}\langle\beta(y(s), P C B y(s)\rangle d s \\
& \leq M+M k\left(t_{0}\right)^{-1}\|P\|^{p} \int_{t_{0}}^{t} k(s)\|y(s)\|^{p} d s+\int_{k\left(t_{0}\right)}^{k(t)} \tilde{N}(\mu) \mu d \mu
\end{aligned}
$$


where

$$
\tilde{N}(t)=\left\{\begin{array}{lll}
-\left(s_{\min }(P)\right)^{\frac{p-2}{2}} \frac{1}{2} N(t) & \text { if } & N(t) \geq 0 \\
-\|P\|^{\frac{p-2}{2}} \frac{1}{2} N(t) & \text { if } & N(t)<0
\end{array}\right.
$$

$s_{\text {min }}(P)$ denotes the smallest singular value of $P$. Now the right hand side becomes negative by the property of the Nussbaum function which contradicts the positivity of the left hand side. This completes the proof.

That the feedback law $u(t)=-N(k(t)) y(t)$, where $N(\cdot)$ is a Nussbaum gain, together with an appropriate adaptation law leads to a universal adaptive stabilizer for the class (3.12) has been shown for

- $m=1$ and $\dot{k}(t)=y(t)^{2}$ by Willems and Byrnes (1984)

- $m \geq 1$ and introducing a general function $N(k)$ by Mărtensson (1986)

- $m \geq 1$ and more general adaptation laws and switching functions by Owens et al. (1989)

Ioannou (1986) considered systems belonging to (3.12) which are coupled with a 'parasitic slow' linear system. He showed that under certain assumptions the Willems-Byrnes controller (2.9) is a universal adaptive stabilizer if the initial state of the unknown system lies in a certain bounded region.

An alternative approach to Nussbaum's switching strategy makes use of the following switching decision function which determines the switching tines $0=$ $t_{0}<t_{1}<\ldots$ of the switching function $N: \mathbb{R}_{+} \rightarrow\{-1,+1\}$ in the following way. Consider the switching decision function $\psi(\cdot)$

$$
\psi(t):= \begin{cases}k(0), & \int_{0}^{t} y^{2}(\tau) d \tau=0 \\ \int_{0}^{t} N(\tau) k(\tau) y^{2}(\tau) d \tau\left[\int_{0}^{t} y^{2}(\tau) d \tau\right]^{-1}, & \int_{0}^{t} y^{2}(\tau) d \tau \neq 0\end{cases}
$$

with $\dot{k}=y^{2}$. If $\left\{\lambda_{i}\right\}_{i \in N}$ is a strictly increasing, unbounded sequence of real, positive numbers or 'thresholds', then $N(t)$ is defined by the following algorithm:

$$
i=0
$$

$$
N\left(t_{i}\right):=1
$$

$$
\begin{aligned}
& t_{i+1}:=\min \left\{t>t_{i} \mid N\left(t_{i}\right) \psi(t)<\lambda_{i+1} k\left(t_{0}\right)\right\} \\
& N(t):=N\left(t_{i}\right), t \in\left[t_{i}, t_{i+1}\right) \\
& N\left(t_{i+1}\right):=-N\left(t_{i}\right) \\
& i:=i+1 \\
& \text { go to } \quad(*)
\end{aligned}
$$

The algorithm is well-defined because

(i) $\psi(t)$ is monotonic on any interval $t>0$ where $N(t)$ is constant

(ii) $\psi\left(t_{0}\right)=k\left(t_{0}\right)$ ensures correct initialization of the algorithm. 
Using the ideas presented in Ilchmann and Owens (1991), the following theorem can be shown. Note that the switching parameter $N(k)$ is adjusted in finite time and that it is in the hand of the designer to choose an appropriate sequence of thresholds.

THEOREM 3.10. The controller

$$
u(t)=-N(k(t)) k(t) y(t), \quad \dot{k}(t)=\|y(t)\|^{p}, \quad k(0) \in \mathbb{R}
$$

where $N(\cdot)$ is produced by (3.15), is a universal adaptive stabilizer for the class (3.12). Moreover, $\psi(t)$ has a finite limit $\psi_{\infty}$ as $t \rightarrow+\infty$, and the switching function $N(t)$ switches only a finite number of times $t_{1}, t_{2}, \ldots, t_{M}$, so that $N(t)$ is constant for $t \geq t_{M}$.

\section{4. $\operatorname{det}(C B) \neq 0$}

If it is only known that the system has an invertible high frequency gain but the spectrum is mixed, i.e. $\operatorname{det}(C B) \neq 0$, then the construction of a universal adaptive stabilizer is based on the following result from linear algebra proved by Mårtensson [5], Section 8 .

LEMMA 3.11. There exists a finite set $\left\{K_{1}, \ldots, K_{N}\right\} \subset G L_{m}(\mathbb{R})$ with the property that, for any $M \in G L_{m}(\mathbb{R})$, there exists $i \in \underline{N}$ such that $\sigma\left(M K_{i}\right) \subset \mathbb{C}_{-}$.

Now the feedback law is given by

$$
u(t)=k(t) K_{S(k(t))} y(t)
$$

where

$$
\begin{gathered}
S: \mathbb{R} \rightarrow\{1, \ldots, N\}=\underline{N} \\
S(k)=\left\{\begin{array}{ll}
1 & \text { if } k \in\left(-\infty, \tau_{1}\right) \\
i & \text { if } k \in\left[\tau_{l N+i}, \tau_{l N+i+1}\right)
\end{array} \text { for some } \quad l \in \mathbb{N}_{0}, i \in \underline{N}\right.
\end{gathered}
$$

is a switching function driven by $k(t)$ so that $K_{S(k(t))}$ cycles through the spectrum unmixing set $\left\{K_{1}, \ldots, K_{N}\right\}$ and $\left\{\tau_{i}\right\}_{i \in \mathbb{N}}$ is a monotone increasing sequence of switching points which satisfy

$$
\lim _{i \rightarrow \infty} \frac{\tau_{i-1}}{\tau_{i}}=0
$$

The switching sequence necessarily fulfils $\lim _{i \rightarrow \infty} \tau_{i}=\infty$. The class of switching sequences satisfying (3.19) is more restrictive than in the single-input single-output case, since for the sequence $\tau_{i}:=i^{2}(3.19)$ does not hold true. However, $\tau_{i+1}:=\tau_{i}^{2}$ and $\tau_{i+1}:=\tau_{i}+\exp \left\{i^{2}\right\}$ satisfy (3.19).

Under the above assumptions, the following result is available.

THEOREM 3.12. The feedback and adaptation law

$$
u(t)=k(t) K_{S(k(t))} y(t), \quad \dot{k}(t)=\|y(t)\|^{p}, \quad k(0) \in \mathbb{R}
$$

is a universal adaptive stabilizer for the class of multivariable minimum phase systems of the form (3.1) which satisfy $\operatorname{det}(C B) \neq 0$. 
The intuition behind this control strategy is similar to Nussbaum's idea. If the 'correct' $K_{i}$ is hit, the gain is large enough, and the time interval until the next possible switch is long enough (which is ensured by condition (3.19)), then the system settles down and no more switchings occur.

This result was claimed by Byrnes and Willems (1984) and by Mårtensson (1986). However, both proofs are incomplete, a correct proof is given in Ilchmann and Logemann (1991).

\subsection{Exponential stabilization}

For first-order systems it has been shown, in Section 2, that the trajectory $x(\cdot)$ of the closed-loop adaptive control system (2.5) decays exponentially to zero. It also follows that the terminal system defined by $\dot{z}(t)=\left[a-k_{\infty} b c\right] z(t), k_{\infty}:=$ $\lim _{t \rightarrow \infty} k(t)$ is exponentially stable. This was not shown for higher-order systems, where only asymptotic decay to zero was proved. Note that we did not show that the terminal system

$$
\dot{x}=\left[A-k_{\infty} B C\right] x(t), \quad k_{\infty}=\lim _{t \rightarrow \infty} k(t)
$$

is exponentially stable, but only that each trajectory of the closed-loop system tends to zero asymptotically. Counterexamples where (3.21) is unstable can easily be constructed. However, computer simulations have shown that the controller (3.13) produces in most cases an exponentially stable terminal system. But, to the author's knowledge, it is still an open problem if generically, with respect to the initial conditions $x(0) \in \mathbb{R}^{n}, k(0) \in \mathbb{R}$, the terminal system produced by the universal adaptive controller (3.13) is exponentially stable.

To overcome the lack of exponential decay, it is possible either to strengthen the minimum phase assumption on the system class or to introduce additional dynamics into the adaptation law.

If $(A, b, c)$ is in the class $(2.3)$, then for $\omega>0$ sufficiently small $\left(A+\omega I_{n}, b, c\right)$ belongs also to (2.3). If the adaptation mechanism is chosen to ensure that $x_{\omega}(\cdot)$ is an asymptotically stable (and hence bounded) solution of the closed-loop system

$$
\dot{x}_{\omega}(t)=\left[\left(A+\omega I_{n}\right)-k(t) b c\right] x_{\omega}(t)
$$

then the solution of

$$
\dot{x}(t)=[A-k(t) b c] x(t),
$$

given by $x(t)=e^{-\omega t} x_{\omega}(t)$, must be of exponential decay. Examples of such adaptation mechanisms are the so-called 'exponentially weighted' controllers

$$
k(t)=k_{0}+\max _{0 \leq s \leq t} e^{\omega s}\|y(s)\|, \quad \text { see Owens et al. (1987) }
$$

and

$$
\dot{k}(t)=e^{\omega t}\|y(t)\|^{2}, \quad \text { see Logemann (1990) }
$$

which consequently yield the desired stabilization result. However, it does require knowledge of a suitable value of $\omega$. 
In order to apply the strategy explained above, one possibility is (see Logemann (1990)) to strengthen the minimum phase condition defining the system class $\Sigma$ to satisfy

$$
\operatorname{det}\left[\begin{array}{cc}
s I_{n}-A & B \\
C & 0
\end{array}\right] \neq 0 \text { for all } s \in\{\lambda \in \mathbb{C} \mid \operatorname{Re} \lambda>-\omega\}
$$

for some known $\omega>0$.

Another possibility is to consider schemes that adaptively find a suitable value for $\omega$ on-line. This idea was introduced for a special control law in Ilchmann and Owens (1990), where it has been shown that exponential stabilization can be achieved by choosing $\omega$ adaptively using the control scheme defined by

$$
\dot{k}(t)=e^{2 \omega(t) t}\|y(t)\|^{2}, \quad k(0)>-1, \quad \omega(t)= \begin{cases}1 & \text { for } t \in[0, h) \\ \frac{1}{1+k(t-h)} & \text { for } t \geq h\end{cases}
$$

where $h \geq 0$ is arbitrary. The idea behind this is the knowledge that, for some $\omega^{*}>0$, the adaptive control law $\dot{k}(t)=\exp \left(2 \omega^{*} t\right)\|y(t)\|^{2}$ will exponentially stabilize the system. Thus, as long as $\omega(t)$ is too large, $x(t)$ will increase and the gain grows whence $\omega(t)$ becomes smaller. Eventually $\omega(t)$ is small enough to guarantee convergence of $k(t)$. Now it follows from (3.25) that $\omega(t)$ converges itself.

In fact, the example (3.25) can be extended since we only use that $\omega: \mathbb{R}_{+} \rightarrow \mathbb{R}_{+}$ is a continuously differentiable function which satisfies the conditions

$$
\left.\begin{array}{rl}
\omega(k) & \text { is non-increasing in } k \in \mathbb{R}_{+} \\
\omega(k) & >0 \text { for all } k \in \mathbb{R}_{+} \text {if } \omega(\cdot) \neq 0 \\
\lim _{k \rightarrow \infty} \omega(k) & =0
\end{array}\right\}
$$

'This puts us into a position to prove a more general result.

Theorem 3.13. Suppose $N(\cdot)$ is a Nussbaum gain. Then the feedback law

$$
u(t)=N(k(t)) y(t) \quad(\text { respectively, } \quad u(t)=-\operatorname{sgn}(C B) y(t))
$$

and the adaptation law

$$
\dot{k}(t)=e^{2 \omega(t) t}\|y(t)\|^{2}, \quad k(0)>-1, \quad \omega \text { satisfies (3.26) }
$$

is a universal adaptive stabilizer for the class (2.1) (respectively, (3.5)), which produces an exponentially decaying solution of the closed-loop system.

A proof is given in Ilchmann and Owens (1990). A version for the non-differential gain adaptation using the switching decision function (3.14) is presented in Ilchmann and Owens (1991, 1991a). If $\dot{k}(t)$ in Theorem 3.12 is substituted by (3.25), then exponential decay of the solution of the closed-loop system holds true. This has been proved by Ilchmann and Logemann (1991).

Unfortunately, all contributions described in this subsection have the disadvantage that the gain adaptation $y \mapsto k$ is achieved by an unbounded function. For a more satisfying approach see Section 5.1. 


\subsection{Tracking}

In this section, we consider the tracking problem for the following class of multiinput, multi-output, linear, minimum phase systems

$$
\left.\begin{array}{l}
\dot{x}(t)=A x(t)+B u(t), \quad y(t)=C x(t), \quad x(0) \in \mathbb{R}^{n} \\
(A, B, C) \in \mathbb{R}^{n \times n} \times \mathbb{R}^{n \times m} \times \mathbb{R}^{m \times n}, \quad \operatorname{det}(C B) \neq 0 \\
(A, B, C) \text { is minimum phase, } n \text { arbitrary }
\end{array}\right\}
$$

and the class of reference signals

$$
\mathcal{Y}_{\text {ref }}:=\left\{y_{\text {ref }} \in \mathcal{C}^{\infty}\left(\mathbb{R}, \mathbb{R}^{m}\right) \mid \alpha\left(\frac{d}{d t}\right) y_{r e f}(t) \equiv 0\right\}
$$

where $\alpha(s) \in \mathbb{R}[s]$ is a monic polonomial with zeros in $\overline{\mathbb{C}}_{+}$only. Note that $0 \in \mathcal{Y}_{\text {ref }}$, therefore it is not relevant to consider the case that $\alpha(s)$ has zeros in $\mathbb{C}_{-}$since the corresponding modes are decaying exponentially.

One possibility of handling this problem is to make use of the internal model principle, that is, a reduplicated model of the dynamic reference signals is incorporated as a precompensator in the feedback loop, see Wonham (1979), Section 8.8. For a different approach, see Section 4.3 and 5.2. Here, the precompensator is chosen as follows. Let $\beta(s) \in \mathbb{R}[s]$ be a monic Hurwitz polynomial of degree $p=\operatorname{deg}(\alpha)$, and choose a minimal realization of $\beta(s) / \alpha(s)$, denoted by $(\hat{A}, \hat{B}, \hat{C}, I) \in \mathbb{R}^{p \times p} \times \mathbb{R}^{p} \times \mathbb{R}^{1 \times p} \times \mathbb{R}$, and the precompensator is given by

$$
\dot{\xi}(t)=\hat{A}^{*} \xi(t)+\dot{B}^{*} v(t), \quad u(t)=\hat{C}^{*} \xi(t)+v(t), \quad \xi(0) \in \mathbb{R}^{m}
$$

where

$$
\begin{aligned}
& \hat{A}^{*}=\operatorname{diag}\{\hat{A}, \ldots, \hat{A}\} \in \mathbb{R}^{m p \times m p}, \quad \hat{B}^{*}=\operatorname{diag}\{\hat{B}, \ldots, \hat{B}\} \in \mathbb{R}^{m p \times m}, \\
& \hat{C}^{*}=\operatorname{diag}\{\hat{C}, \ldots, \hat{C}\} \in \mathbb{R}^{m \times m p} .
\end{aligned}
$$

Then the input-output behaviour $v \mapsto y$ of the series interconnection formed by (3.27) and (3.29) is described by

$$
\dot{\bar{x}}(t)=\bar{A} \bar{x}(t)+\bar{B} v(t), \quad y(t)=\bar{C} \bar{x}(t), \quad \dot{x}(0) \in \mathbb{R}^{n+m p}
$$

where

$$
\bar{A}=\left[\begin{array}{cc}
A & B \hat{C}^{*} \\
0 & \hat{A}^{*}
\end{array}\right], \quad \bar{B}=\left[\begin{array}{c}
B \\
\hat{B}^{*}
\end{array}\right], \quad \bar{C}=[C, 0], \quad \bar{x}=\left[\begin{array}{l}
x \\
\xi
\end{array}\right]
$$

In order to rewrite this as a stabilization problem, the following two lemmata are needed.

LEMMA 3.14. (A,B,C) belongs to (3.27) if and only if $(\bar{A}, \bar{B}, \bar{C})$ belongs to (3.27) and $C B=C B$.

LEMMA 3.15. For every $y_{\text {ref }} \in \mathcal{Y}_{\text {ref }}$ there exists a $\tilde{x}_{0} \in \mathbb{R}^{n+m p}$ such that

$$
y_{r e f}(t)=\bar{C} \tilde{x}(t), \quad \dot{\tilde{x}}(t)=\bar{A} \tilde{x}(t), \quad \tilde{x}(0)=\tilde{x}_{0}
$$




$$
\begin{aligned}
& \text { Now, } x_{t}(t):=\bar{x}(t)-\bar{x}(t) \text { satisfies } \\
& \dot{x}_{t}(t)=A x_{t}(t)+B v(t), \quad y_{r \in f}(t)-y(t)=C \bar{x}_{t}(t), \quad x_{e}(0)=\bar{x}(0)-\tilde{x}(0) .
\end{aligned}
$$

This yields the following theorem.

THEORLM 3.16 . If

$$
u(t)=f(k(t), y(t)), \quad \dot{k}(t)=g(k(t), y(t)), \quad k(0) \in \mathbb{R}
$$

is a universal adaptive stabilizer for the class (3.27), then

$$
\begin{aligned}
& v^{\prime}(t)=y_{r e f}(t)-y(t) \\
& v(t)=f(k(t), c(t)), \quad \dot{k}(t)=g(k(t),(t)), \quad k(0) \in \mathbb{K} \\
& u(t)=C^{*} \xi(t)+D^{*} v(t), \quad \dot{\xi}(t)=\dot{A}^{*} \xi(t)+B^{*} v(t), \quad \xi(0) \in \mathbb{P}^{m p}
\end{aligned}
$$

is a universal adapline tracking controller for the class (3.27) and the class of refcrence signals $\mathcal{Y}_{\text {ref }}$ given by (3.27).

The previous presentation is given by Miller and Davison (1991b), and independently by 'lownley and Owens (1991). The results in Miller and Davison (1991b) cover a more general form including $m \geq p$ to some extent, ccrtain disturbances $w$ satisfying $\alpha\left(\frac{d}{d t}\right) w=0$ are allowed in the state and output equation, and they show that internal stability is preserved in the sense that $x$ and $u$ do not blow up faster than $y_{r e f}$.

For the special case of single-input, single output, minimum phase systems of relative degree 1 , with $\alpha(s)$ having roots in $\bar{C}_{-}$of multiplicity one if they are on $i \mathbb{R}$, the same approach has been used by Helmke ot al. (1990) to present a universal adaptive tracking controller.

Mareels (1984) was the first who used the internal model principle to construct a universal adaptive tracking controller for single-input single-output systems of relative degree $p \geq 1$, sec Section 4.3 .

Tao and Ioannou (1991) have introduced the following different tracking controller for single-input single-output systems.

Proposition 3.17. The adaptation law

$$
\left.\begin{array}{l}
\dot{\theta}(t)=\omega(t)\left[y_{r e f}(t)-y(t)\right], \quad \theta(0) \in \mathbb{R}^{2(l+1)} \\
\dot{k}(t)=\langle\theta(t), \omega(t)\rangle\left[y_{r e f}(t)-y(t)\right], \quad k(0) \in \mathbb{R}
\end{array}\right\}
$$

where $\omega(t)=\left[y_{\text {ref }}(t)-y(t), 1, \sin \omega_{1} t, \ldots, \sin \omega_{l} t, \ldots, \cos \omega_{1} t, \ldots, \cos \omega_{l} t\right]^{T}$, together with the feedback law

$$
u(t)=k(t)^{2} \sin k(t) \cdot\langle\theta(t), \omega(t)\rangle
$$

is a universal adaptive tracking controller for the class (2.3) and the class of reference signals consisting of

$$
\left.\begin{array}{l}
y_{r e f}(t)=a_{0}+\sum_{i=1}^{l} a_{i} \sin \omega_{i} t+b_{i} \cos \omega_{i} t \\
\omega_{1}, \ldots, \omega_{l} \in \mathbb{R} \quad \text { are known, } a_{0}, \ldots, a_{l}, b_{1}, \ldots, b_{l} \in \mathbb{R} \text { are unknown. }
\end{array}\right\}
$$

'The proof by Tao and Ioannou (1991) is not based on a conversion to an adaptive stabilization problem. 


\subsection{Robustness}

Robustness for the adaptive controllers surveyed in the previous sections has been considered for state and input nonlinearities entering a system belonging to (3.1) in the following form

$$
\begin{array}{rr}
\dot{x}(t) & =A x(t)+\varphi(t, x(t))+d(t)+B\left[u(t)+\eta_{1}(t, x(t))\right. \\
y(t) & \left.=C x(t) \quad+\eta_{2}(t, x(t))+\eta_{3}(t, x(t), u(t))\right]
\end{array}
$$

and also for sector-bounded input and output nonlinearities $\xi$ and $\zeta$ so that the real input $\hat{u}$ can enter the system via $u(t)=\xi(t, \hat{u}(t))$ and the real output measurement is given by $y(t)=\zeta(t, y(t))$. All nonlinear functions are appropriately defined in order to ensure uniqueness and no finite escape time, we omit details for brevity.

The term $\varphi(t, x)$ represents time-varying state depending perturbations which are assumed to be of sufficinetly small finite gain, thus proving well posedness. $d(t)$ represents an arbitrary $L_{p}(0, \infty)$ function. The time-varying input perturbation are of bounded growth or can be unbounded if they are of 'correct' sign. More precisely, the following results have been achieved.

Helmke and Prätzel-Wolters (1988) showed that the Willems-Byrnes controller (2.9) for $N(k) \equiv-\operatorname{sgn}(c b)$ is a universal adaptive stabilizer if for all $(t, x) \in \mathbb{R}^{2}$ we have

$$
|\varphi(t, x)| \leq \hat{\varphi}|x| \text { for some (unknown) } \hat{\varphi} \geq 0
$$

and $d(t)$ is an $L_{p}(0, \infty)$ function.

An improvement of the local behaviour of the controller (2.9) is case of known high frequency gain is achieved by Cabrera and Furuta (1989) who modify the adaptation law in (2.9) to $\dot{k}=-\sigma k+y^{2}$ for some $\sigma>0$. Under certain assumptions on the system class the closed loop system is robust against bounded disturbances.

Theorem 3.7 holds true for multivariable systems if for all $(t, x, u) \in \mathbb{R} \times \mathbb{R}^{n} \times \mathbb{R}^{m}$ and some (unknown) $\hat{\varphi}, \hat{\eta}_{1}, \hat{\eta}_{2}, \hat{\eta}_{3} \geq 0$ we have

$$
\begin{aligned}
\|\varphi(t, x)\| & \leq \hat{\varphi}\|x\|, \quad \hat{\varphi} \text { sufficiently small } \\
\left\|\eta_{1}(t, x)\right\| & \leq \hat{\eta}_{1}\|x\| \\
y(t)^{T} C B \eta_{2}(t, x) & \leq 0 \\
y(t)^{T} C B \eta_{3}(t, x, u) & \leq\|y\| \hat{\eta}_{3}[\|x\|+\|u\|]
\end{aligned}
$$

see Owens et al. (1987).

In the single-input, single-output case Theorem 3.9 is valid, if for all $(t, x) \in$ $\mathbb{R} \times \mathbb{R}^{n}$ and some (unknown) $\hat{\eta}_{1}, \hat{\eta}_{2} \geq 0$, we have

$$
\left\|\eta_{1}(t, x)\right\| \leq \hat{\eta}_{1}\|x\|, \quad y(t)^{T} C B \eta_{2}(t, x) \leq 0
$$

This has been proved in Prätzel-Wolters et al. (1989).

Theorem 3.9 holds also true if the Nussbaum function is scaling invariant and if the class of single-input single-output systems is subjected to actuator and sensor nonlinearities $\xi(t, \hat{u})$ and $\zeta(t, y)$ which are sector-bounded, i.e. for all $t \in \mathbb{R}$ and some (unknown) $\beta \geq \alpha>0$ we have

$$
\alpha \hat{u} \leq \xi(t, \hat{u}) \leq \beta \hat{u} \quad \text { for all } \hat{u} \in \mathbb{R}_{+}, \quad \beta \hat{u} \leq \xi(t, \hat{u}) \leq \alpha \hat{u} \text { for all } \hat{u} \in \mathbb{R}_{-}
$$


and for $\zeta$ analogously. This is a consequence of a general result for retarded systems proved by Logemann (1990). Ilchmann and Owens (1991a) have shown that Theorem 3.10 remains valid for single-input single-output systems in case of sectorbounded input nonlinearities and that exponential stabilization, as presented in Theorem 3.13, is also possible if a switching decision function is used for the gain adaptation mechanism.

Theorem 3.12, proved by Ilchmann and Logemann (1991), and the extension to achieve exponential stabilization, as stated in 'Theorem 3.13 , remain valid in the presence of nonlinearities satisfying (3.37) and (3.38).

Certain well-posedness properties are claimed by Tao and loannou (1991) if the controller (3.33), (3.34) is modified so that discontinuous feedback is used. However, discontinuities on the right hand side of the closed-loop differential equation is not taken into account in their proof.

\section{Minimum phase systems of higher relative degree}

\subsection{Observer-based model reference controllers}

In this section, we report some results which show the feasibility of the adaptive control problem, the controllers are complicated in nature. It has been a longstanding problem to prove that an adaptive controller not only stabilizes or tracks the output but ensures stability of the internal variables as well, i.e. an adaptive controller in the sense of Definition 1.1.

Feuer and Morse (1978) introduced an adaptive stabilizer for single-input singleoutput systems satisfying the assumptions (A1) (A4). This has been, to the author's knowledge, the first proof of the global stability behaviour of an adaptive controller. The controller is not based on any identification algorithm and does not use a sufficiently rich probing signal: however, it uses a full state observer controller and is complicated in nature. Its dimension grows rapidly with increasing relative degree of the systems allowed in the system class. Feuer and Morse (1978) also solved the adaptive model reference (respectively, tracking) problem for the system class satisfying (A1)-(A4), the class of reference signals produced by reference models consisting of controllable and observable systems of the form $\left(A_{r}, b_{r}, c_{r}\right)$, where $A_{r}$ is exponentially stable and the relative degree of the transfer function has to be greater or equal to the relative degree of the process transfer function, and with piecewise constant and bounded input signals.

This controller has been simplified by Morse (1980), and Morse (1984) could remove the known sign assumption (A1) by making use of a switching function as introduced by Nussbaum (1983), cf. Section 2. 'The same class of reference models and reference signals is considered. Robustness has not been investigated. However, the relative degree is only allowed to be 1 or 2 and has to be known.

These results have been generalized to relative degree 1 or 2 systems, where the degree is unknown, in Morse (1987), see also Morse (1988a). The model reference adaptive control problem has been solved for a certain class of reference models, however an upper bound on the state dimension of the system has to be known. Extensions of these results for the case where the systems is of arbitrary but known relative degree $n^{*}$, the reference model has relative degree $n^{*}-1$, are presented in 
Mudgett and Morse (1985).

However, all controllers in the above references are still based on the one presented in Morse (1980) and thus complicated in nature.

An alternative stabilizer which is valid for single-input single-output systems satisfying (A1)-(A4) has been introduced by Narendra et al. (1980), however it is not simpler.

\subsection{Non-observer-based stabilization and tracking}

Before we survey some results on minimum phase systems of relative degree $p \geq 2$, we try to give an intuition for the design of an adaptive stabilizer.

Consider the class of single-input single-output, minimum phase, relative degree 2 systems with positive high frequency gain, i.e. systems of the form

$$
\left.\begin{array}{l}
\dot{x}(t)=A x(t)+b u(t), \quad y(t)=c x(t), \quad x(0) \in \mathbb{R}^{n} \\
(A, b, c) \in \mathbb{R}^{n \times n} \times \mathbb{R}^{n} \times \mathbb{R}^{1 \times n} \quad \text { is minimum phase, } c b=0, c A b>0 .
\end{array}\right\}
$$

It is well known that there exist two dimensional controllable and observable minimum phase systems of relative degree 2, which cannot be stabilized by feedback of the form $u=-k y$, see Example 6.2.1 in Sontag (1990). However, if feedback of the derivative of the output is allowed, then the closed loop system is of relative degree 1 and standard techniques can be applied. This is demonstrated in the following proposition, which is not available in the literature.

Proposition 4.1. The feedback law

$$
u(t)=-\left[k(t)+|y(t)|^{p}\right] y(t)-k(t) \dot{y}(t)=-\left[k(t) y(t)+\frac{d}{d t}(k(t) y(t))\right]
$$

together with the adaptation rule

$$
\dot{k}(t)=|y(t)|^{p}, \quad k(0) \in \mathbb{R}, \quad p \geq 1
$$

applied to any system of the class (4.1) yields a closed-loop system with a bounded solution on $\mathbb{R}_{+}$, and the properties $\lim _{t \rightarrow \infty} x(t)=0, \lim _{t \rightarrow \infty} k(t) \in \mathbb{R}$ exists.

Proof. The feedback system can be rewritten as a system of relative degree 1 as follows. Inserting the feedback (4.2) into a system $(A, b, c)$ yields

$$
\dot{x}(t)=A x(t)-b k(t) y(t)-\frac{d}{d t}(b k(t) y(t)), \quad x(0) \in \mathbb{R}^{n} .
$$

Introducing the new variable $v(t)=x(t)+b k(t) y(t)$ leads to the closed-loop system

$$
\left.\begin{array}{l}
\dot{v}(t)=A v(t)-(I+A) b k(t) y(t), \quad y(t)=c v(t) \\
v(0)=x(0)+b k(0) y(0) \\
\dot{k}(t)=\|y(t)\|^{p}, \quad k(0) \in \mathbb{R}
\end{array}\right\}
$$

Since $c(I+A) b=c A b$, the relative degree of the system in the first equation in $(4.5)$ is 1 and the minimum phase property is preserved because of

$$
\left[\begin{array}{cc}
s I-A & (I+A) b \\
-c & 0
\end{array}\right]\left[\begin{array}{ll}
I & b \\
0 & 1
\end{array}\right]=\left[\begin{array}{cc}
s I-A & b \\
-c & 0
\end{array}\right]\left[\begin{array}{cc}
I & 0 \\
0 & s+1
\end{array}\right]
$$


Now, Theorem 3.6 gives $\lim _{t \rightarrow \infty} v(t)=0$ and $\lim _{t \rightarrow \infty} k(t)$ exists, and hence

$$
\lim _{t \rightarrow \infty} x(t)=0 .
$$

A generalization of this result to higher-relative-degree systems is possible if we assume that higher derivatives are available for feedback, a clever introduction of an internal variable $v$ is then due to a trick of Miller and Davison (1991). However, if $\dot{y}$ is not available, an approximation of $\dot{y}$, respectively a dynamic compensator, will be used. For systems belonging to the class (4.1) it can be shown that the dynamic compensator

$$
\hat{u}(s)=-k^{3} \frac{s+1}{s+k^{2}} \hat{y}(s), \quad \text { for constant } \quad k \in \mathbb{R}
$$

yields exponential stability of the closed loop system (4.1), (4.6) if $k$ is sufficiently large. Rewriting (4.6) as

$$
\hat{u}(s)=-(s+1)[k \hat{\theta}(s)], \quad \hat{\theta}(s):=\frac{k^{2}}{s+k^{2}} \hat{y}(s)
$$

leads to the following guess of the adaptive compensator in the time domain

$$
u(t)=-\left[k(t) \theta(t)+\frac{d}{d t}(k(t) \theta(t))\right], \quad \dot{\theta}(t)=-k(t)^{2} \theta(t)+k(t)^{2} y(t), \quad \theta(0) \in \mathbb{R} .
$$

In fact, if we consider the solution $\theta_{k}(\cdot)$ of the second equation in (4.8) for fixed $k(\cdot) \equiv k \in \mathbb{R}$, then $\lim _{k \rightarrow \infty}\left(\theta_{k}-y\right)_{L_{1}}=0$. Thus $\theta(t)$ is an approximation of $y(t)$ if $k(t)$ is big enough. We obtain the following result.

I'HEOREM 4.2. The adaptation rule $k(t)=y(t)^{2}, k(0) \in \mathbb{R}$, together with (4.8) is a universal adaptive stabilizer of order 2 for the class of systems (4.1).

Proof. Suppose $(A, b, c)$ belongs to the class (4.1) and is transformed as in (4.5). An application of Proposition 3.2 allows to rewrite the closed loop system as

$$
\frac{d}{d t}\left(\begin{array}{c}
\theta(t) \\
y(t) \\
z(t)
\end{array}\right)=\left[\begin{array}{ccc}
-k(t)^{2} & k(t)^{2} & 0 \\
-c A b k(t) & A_{1} & A_{2} \\
0 & A_{3} & A_{4}
\end{array}\right]\left(\begin{array}{c}
\theta(t) \\
y(t) \\
z(t)
\end{array}\right)
$$

where $A_{4} \in \mathbb{H}^{(n-1) \times(n-1)}$ is exponentially stable and $A_{1} \in \mathbb{R}^{\mathbb{R}}, A_{2}^{T}, A_{3} \in \mathbb{R}^{n-1}$. The coordinate transformation $\xi:=\theta-y$ leads to

$$
\frac{d}{d t}\left(\begin{array}{c}
\xi(t) \\
y(t) \\
z(t)
\end{array}\right)=\left[\begin{array}{ccc}
-k(t)^{2}-c A b k(t) & -\left(c A b k(t)+A_{1}\right) & -A_{2} \\
-c A b k(t) & c A b k(t)+A_{1} & A_{2} \\
0 & A_{3} & A_{4}
\end{array}\right]\left(\begin{array}{c}
\xi(t) \\
y(t) \\
z(t)
\end{array}\right)
$$

Let $P$ be the unique positive definite solution of $P A_{4}+A_{4}^{T} P=-I$ and consider the Lypunov function candidate

$$
V(\xi, y, z):=\frac{1}{2} \xi^{2}+\frac{1}{2} y^{2}+\langle z, P z\rangle
$$


Then the derivative of $V$ along the solution of (4.10) is for a suitable constant $K>0$

$$
\dot{V}(t) \leq[-c A b k(t)+K] y(t)^{2}
$$

and integration yields

$V(t) \leq V(0)+\int_{0}^{t}[-c A b k(s)+K] y(s)^{2} d s=V(0)-c A b \int_{k(0)}^{k(t)} \mu d \mu+K[k(t)-k(0)]$

where we made use of the substitution $k(s)=\mu$. If $k(t)$ is unbounded, the right hand side of (4.11) becomes negative, hence producing a contradiction. The remainder of the proof uses similar arguments as in Theorem 3.6 .

Byrnes and Isidori (1986) gave a different (and incomplete) proof of Theorem 4.2.

For higher relative degree minimum phase systems, the intuition arises from the non-adaptive case as well. It is shown in Martensson (1986) that the linear time-invariant compensator

$$
\hat{u}(s)=-k^{2 p-1} \frac{(s+1)^{p-1}}{\left(s+k^{2}\right)\left(s+k^{4}\right) \ldots\left(s+k^{2^{p-1}}\right)} \hat{y}(s)
$$

stabilizes a minimum phase systems with positive high frequency gain and relative degree less or equal to $p$ for $k$ sufficiently large. The same result has been shown by Khalil and Saberi (1987) for the different compensator

$$
\hat{u}(s)=-k^{2 p-1} \frac{(s+1)^{p-1}}{\left(s+k^{2}\right)^{p-1}} \hat{y}(s) .
$$

Then the problem is to determine a suitable adaptive controller in the time domain. Note that the transformation from (4.7) to (4.8) is meaningless if $k(\cdot)$ is depending on $t$. However, the Lyapunov function candidate sometimes gives a hint for the correct time-domain realization.

A very early contribution to solve a certain adaptive tracking problem was made by Mareels (1984). He considers the following class of single-input single-output systems with arbitrary but known relative degree, known sign of the high frequency gain, and known upper bound for its magnitude.

$$
\left.\begin{array}{l}
\dot{x}(t)=A x(t)+b u(t), \quad y(t)=c x(t), \quad x(0) \in \mathbb{R}^{n} \\
c b=c A b=\ldots=c A^{p-2} b=0, \quad 0<c A^{p-1} b<g_{0} \\
(A, b, c) \in \mathbb{R}^{n \times n} \times \mathbb{R}^{n} \times \mathbb{R}^{1 \times n}, \quad n \text { is unknown, } p, g_{0} \text { are known }
\end{array}\right\}
$$

As opposed to the approaches mentioned in Section 4.1, Mareels' controller does not use a state observer, instead it is relatively simple and uses ideas partially presented above. His approach is based on the high-gain properties of the system class. For the sake of simplicity we consider systems of relative degree 2 . The following lemma is basic for the construction of the adaptive stabilizer.

LEMMA 4.3. Suppose $\left(A_{i}, b_{i}, c_{i}\right) \in \mathbb{R}^{i \times i} \times \mathbb{R}^{i} \times \mathbb{R}^{1 \times i}$ are controllable and observable systems with exponentially stable $A_{i}, i=1,2$, so that the polynomial

$$
g_{0}+c_{1} b_{1} s+c_{2} b_{2} s^{2}+s^{3}
$$


is Hurwitz. (Note that $g_{0}$ is the upper bound of the magnitude of the high frequency gain.). Consider the following dynamical system described by

$$
\begin{gathered}
u(t)=k\left[k^{2}\left(c_{1}, c_{2}\right)\left(\begin{array}{l}
x_{1}(t) \\
x_{2}(t)
\end{array}\right)+k^{2} y(t)\right] \\
\frac{d}{d t}\left(\begin{array}{c}
x_{1}(t) \\
x_{2}(t)
\end{array}\right)=\left[\begin{array}{cc}
A_{1}+k b_{1} c_{1} & k b_{1} c_{2} \\
k^{2} b_{2} c_{1} & A_{2}+k^{2} b_{2} c_{2}
\end{array}\right]\left(\begin{array}{l}
x_{1}(t) \\
x_{2}(t)
\end{array}\right)+\left(\begin{array}{c}
k b_{1} \\
k^{2} b_{2}
\end{array}\right) y(t)
\end{gathered}
$$

If $(A, b, c)$ is an element of the class (4.14) for $p=2$ and the feedback compensator (4.15), (4.16) is applied to $(A, b, c)$ then there exists a $k_{0}>0$ such that the closed loop system is exponentially stable for all $k \geq k_{0}$.

The adaptive version of the previous lemma is as follows.

THEOREM 4.4. Let $p=2$. Then the feedback compensator (4.15), (4.16) together with the adaptation law

$$
\frac{d}{d t}\left(k(t)^{2}\right)=f(y(t)), \quad k(0) \in \mathbb{R}
$$

is a universal stabilizer for the class (4.14), provided $f: \mathbb{R} \rightarrow \mathbb{R}$ satisfies for some $F^{\top}>0$ and for all $0<\left|z_{1}\right| \leq\left|z_{2}\right|$

$$
0<f\left(z_{1}\right) \leq f\left(z_{2}\right)<F \quad \text { and } \quad Z \mapsto \int_{0}^{Z}(f(z) / z) d z \quad \text { is continuous. }
$$

An extension to arbitrary but known relative degree $p \geq 1$ is straightforward. It is also shown by Mareels (1984) that the tracking problem for the class (3.35) can be solved, this is done in the same way as presented in Section 3.6.

Morse (1988) has shown that the Willems-Byrnes controller (2.9) with $N(k) \equiv 1$ also works for the class of single-input single-output, minimum phase systems of relative degree 2 with positive damping rate and positive high frequency gain, i.e. the transfer function $g /\left(s^{2}+a s+b\right)$ satisfies $g>0, a>0$. This result has been extended by Corless $(1988,1991)$ as follows.

THEOREM 4.5. The controller $u(t)=k(t) y(t), \dot{k}(t)=y(t)^{2}$ is a universal adaptive stabilizer for the class of controllable and observable systems $(A, b, c) \in \mathbb{R}^{n \times n} \times$ $\mathbb{R} \times \mathbb{R}^{1 \times n}$ which are uniformly stabilizable via high gain feedback, i.e. there exist $\epsilon$, $k^{*}>0$ (depending on $(A, b, c)$ ) so that

$$
\max _{i \in \underline{n}} \operatorname{Re} \lambda_{i}(A-k b c) \leq-\epsilon \text { for all } k \geq k^{*}
$$

where $\lambda_{i}(A-k b c)$ denotes the eigenvalues of $A-k b c$.

Morse (1988) introduces a stabilizer for the following class of relative 1 or 2 systems where the exact relative degree is unknown.

$$
\begin{aligned}
& \dot{x}(t)=A x(t)+b u(t), \quad y(t)=c x(t), \quad x(0) \in \mathbb{R}^{n} \\
& (A, b, c) \in \mathbb{R}^{n \times n} \times \mathbb{R}^{n} \times \mathbb{R}^{1 \times n}, n \text { is unknown } \\
& c b>0 \text { or if } c b=0 \text { then } \quad c A b>0
\end{aligned}
$$

He has proved the following theorem. 
THEOREM 4.6. The feedback law

$$
u(t)=-k(t) \theta(t)-k(t)^{2} y(t)
$$

together with the adaptation law

$$
\left.\begin{array}{l}
\dot{\theta}(t)=-(k(t)+\lambda) \theta(t)-k(t)^{2} y(t), \quad \theta(0) \in \mathbb{R}, \lambda>0 \\
\dot{k}(t)=y(t)^{2}, \quad k(0) \in \mathbb{R}
\end{array}\right\}
$$

is a universal adaptive stabilizer for the class (4.18).

By increasing the dimension of the compensator by one, Morse (1987a) generalized Theorem 4.6 to the class of relative degree 3 systems.

To relax the known sign condition in the class (4.18), Morse (1985) introduced a two-parameter adaptation law of the following form

$$
\begin{aligned}
k_{y}(t) & =\frac{1}{2} y(t)^{2}+\int_{0}^{t} y(s)^{2} d s, \quad k_{\theta}(t)=\theta(t) y(t)+z_{\theta}(t) \\
\dot{z}_{\theta}(t) & =\lambda \theta(t) y(t)-y(t) N(\|k(t)\|)\left[\theta(t) k_{\theta}(t)+y(t) k_{y}(t)\right] \\
& =\left[\lambda y(t)-N(\|k(t)\|) y(t) k_{\theta}(t)\right] \theta(t)-y(t)^{2} N(\|k(t)\|) k_{y}(t)
\end{aligned}
$$

where $\|k\|:=\sqrt{k_{y}+k_{\theta}}, \lambda>0$, and $N(\cdot)$ is a Nussbaum function, see (2.10). Then the following result is obtained.

THEOREM 4.7. The adaptation law (4.21),(4.22) together with

$$
u(t)=N(\|k(T)\|)\left[\theta(t) k_{\theta}(t)+y(t) k_{y}(t)\right], \quad \dot{\theta}(t)=-\frac{1}{2} \lambda \theta(t)+u(t)
$$

is a universal adaptive stabilizer for the class of minimum phase systems $(A, b, c) \in$ $\mathbb{R}^{n \times n} \times \mathbb{R}^{n} \times \mathbb{R}^{1 \times n}$ of relative degree 1 or $\mathbb{2}$.

To get an intuition for this controller, consider the fact that the output feedback compensator

$$
\hat{y}(s)=\frac{N(\|k\|) k_{y}(s+\lambda)}{s+\lambda-N(\|k\|) k_{\theta}} \hat{u}(s)
$$

yields closed loop stability for a suitable constant $k_{y}, k_{\theta} \in \mathbb{R}$.

Mudgett and Morse (1989) introduced an alternative stabilizer to that given by Mareels (1984) for the class (4.14) and $p=2$.

\subsection{Tracking within a ball}

Miller and Davison (1991) considered a modification of the usual adaptive tracking problem. Instead of forcing the error between the plant output and the reference signal asymptotically to zero, it is desired to force the error to be less than an arbitrarily small prespecified constant after an arbitrarily short prespecified period of time with an arbitrarily short upper bound on the overshoot. More precisely they have studied the following problem. Let

$$
\begin{aligned}
& P C_{\infty}:=\text { the set of piecewise constant bounded functions } f: \mathbb{R} \rightarrow \mathbb{R}^{q} \\
& P C_{\infty}^{1}:=\text { the set of continuous } f \in P C_{\infty} \text { which have derivatives in } P C_{\infty}
\end{aligned}
$$


Problem 4.8 Suppose $\epsilon, \delta, T>0$ are prespecified. Find an adaptation law

$$
\left.\left(y(\cdot), y_{r e f}(\cdot)\right)\right|_{[0, t]} \mapsto k(t)
$$

such that the feedback law $u(t)=k(t)$ applied to any system belonging to a prespecified system class and any $y_{r \in f}(\cdot) \in P C_{\infty}^{1}$ yields for the solution of the closed loop systern and the error

$$
\begin{aligned}
& \qquad e(t):=y_{\text {ref }}(t)-y(t) \\
& \text { (i) }-\epsilon \leq \operatorname{sgn}(e(0)) e(t) \leq \max \{\epsilon, \delta+|e(0)|\} \text { for all } t \in[0, T] \\
& \text { (ii) }|e(t)|<\epsilon \text { for all } t \geq T \\
& \text { (iii) } x(\cdot), u(\cdot) \text { are bounded functions. }
\end{aligned}
$$

The class of systems under consideration is

$$
\left.\begin{array}{l}
\dot{x}(t)=A x(t)+b u(t)+E w_{1}(t), \quad y(t)=c x(t)+F w_{2}(t), \quad x(0) \in \mathbb{R}^{n} \\
E \in \mathbb{R}^{n \times n_{1}}, F \in \mathbb{R}^{1 \times n_{1}},(A, b, c) \in \mathbb{R}^{n \times n} \times \mathbb{R}^{n} \times \mathbb{R}^{1 \times n} \text { minimum phase } \\
w_{1}(\cdot) \in P C_{\infty}, w_{2}(\cdot) \in P C_{\infty}^{1} \text { are arbitrary disturbances. }
\end{array}\right\}
$$

Notice that no assumption is made on the relative degree.

The control strategy is based on a gain adaptation which produces piecewise constant gains $k(t)$. Thus it is different to most of the preceding results, only similar in nature to the results given in Section 3.4. The advantage of a switching type controller which switches between constant gains is that the analysis on an interval where the feedback is constant is relatively simple (because one analyses a linear time-invariant system): it has to be ensured that the intervals between the switching times are long enough in order to give the system time to settle down.

First assume that (4.25) is restricted to the class of relative degree $p$ systems. Khalil and Saberi (1987) have shown that the linear time-invariant compensator

$$
u(s)=-k^{2 p-1} \frac{(s+1)^{p-1}}{\left(s+k^{2}\right)^{p-1}} \hat{e}(s)
$$

stabilizes each single-input single-output, minimum phase, relative degree $p \geq 1$ system of proper high frequency gain sign and $k$ sufficiently large. Miller and Davison (1991) choose as a realization of (4.26)

$$
\left.\begin{array}{rl}
\dot{v}(t) & =k^{2} G v(t)+k^{2} H e(t) \\
r(t) & =k J v(t) \\
u(t) & =\sum_{i=0}^{p-1} \alpha_{i} r^{(i)}(t)
\end{array}\right\}
$$

where

$$
G=\left[\begin{array}{ccccc}
-1 & 1 & 0 & \ldots & 0 \\
0 & -1 & 1 & \ldots & 0 \\
0 & 0 & -1 & \ldots & 0 \\
\vdots & \vdots & \vdots & \ldots & 1 \\
0 & 0 & 0 & \ldots & -1
\end{array}\right] \in \mathbb{R}^{(p-1) \times(p-1)}
$$




$$
H=\left[\begin{array}{c}
0 \\
0 \\
\vdots \\
0 \\
1
\end{array}\right] \in \mathbb{R}^{p-1}, \quad J=\left[\begin{array}{c}
1 \\
0 \\
\vdots \\
0 \\
0
\end{array}\right] \in \mathbb{R}^{p-1}
$$

and the $\alpha_{i}$ 's are defined by

$$
\sum_{i=0}^{p-1} \alpha_{i} s^{i}=(s+1)^{p-1} .
$$

Observe that $u$ can be constructed from $e$ and $v$ without differentiation.

Since it is assumed that the relative degree of $(A, b, c)$ is $p$, it can be shown that there exists a clever coordinate transformation so that the closed loop system (4.25), (4.27) can be rewritten as

$$
\dot{z}(t)=A z(t)+(A+I)^{p-1} b r(t)+w_{1}(t), \quad y(t)=c z(t)+w_{2}(t) .
$$

Since (4.28) is minimum phase and of relative degree one, it is possible to use the convenient form given in (3.3). This enables us to obtain tedious estimates of the solution of the closed loop system (4.28) and to prove the following theorem.

Theorem 4.9. Suppose $\epsilon, \delta, T>0$. Let $k(t)$ depend on time and be given by

$$
k(t)=(-i)^{i^{2}} \text { for } t \in\left[t_{i}, t_{i+1}\right)
$$

where the sequence of switching times is determined in two phases:

(i) $t_{1}=0, \quad k(0)=0$

$t_{i}=\min \left\{t>t_{i-1}|| e(t)|=| e(0) \mid+\delta\left[1-2^{-i}\right]\right.$ or $|e(t)| \leq \frac{1}{2} \epsilon$ or $\left.t=T\left[1-2^{-i}\right]\right\}$

If $\left|c\left(t_{i}\right)\right| \leq \frac{1}{2} \epsilon$ for some $i=i_{0}$, then go to phase (ii),

(ii) $t_{i}=\min \left\{t>t_{i-1}:|e(t)|=\epsilon\left[1-1 / 2^{i-i_{0}+1}\right]\right\}$

Then the feedback law (4.27) together with the adaptation law (4.29) is a universal adaptive tracking controller in the sense of Problem 4.8 for all relative degree $p$ systems of the class (4.25).

In Phase (i) the error is forced to stay within the prespecified amount of overshoot and to become smaller than $\frac{1}{2} \epsilon$ by using the high-gain properties. The so-called 'tuning function' $(-i)^{i}$ in $(4.29)$ can be replaced by a simpler function depending on the relative degree $p$. For example, $k(t)=(-3)^{i}$ will work for the relative degree 1 case.

Miller and Davison (1991) have also introduced a modification of the controller which can cope with additive noise $d(\cdot) \in P C_{\infty}^{1}$ in the output. Necessity of the minimum phase condition is also proved.

Remark 4.10. Theorem 4.9 can be extended to the class (4.25), that is neither knowing the precise relative degree nor an upper bound of the relative degree, by searching the controller dimension according to Marrtensson (1985). 'The switching times have to be modified so that it is ensured that the feedback law (4.27) cycles in an appropriate way through different compensators according to the dimensions $1,1,2,2,1,1,2,2,3,3,1,1 \ldots$ 


\section{Non-minimum phase systems}

In Section 5.1, we present the famous result by Mårtensson (1985, 1986) who introduced an adaptive stabilizer for all systems for which the order of a stabilizing compensator is known. This approach has been improved by Miller and Davison $(1987,1988,1989)$ in order to show certain robustness properties resp. exponential Lyapunov stabilization. In Section 5.2 we show how these stabilizers together with an appropriate internal model can be used to design adaptive tracking controllers solving the servomechanism problem. The results of Miller and Davison (1987, 1991 b, 1989) are presented.

Most of the adaptive controllers introduced in the previous sections were smooth in the sense that the right hand side of the adaption law $\dot{k}=g(k, y)$ and of the feedback law $u=f(k, y)$ depends smoothly on its arguments. The controllers presented in this section are mostly discontinuous since the feedback law consists of a switching gain type controller, that means the feedback gain switches at discrete points of time and between these points $k$ is held constant. Therefore, the resulting closed-loop system is a coupling of a piecewise time-invariant linear system and a nonlinear scalar equation: this simplifies the analysis to some extent. This approach is more successful than the 'smooth approach' in the sense that many results have first been shown by using discontinuous feedback controllers. Although in a second step the discontinuities can be smoothed out, cf. Martensson (1986), Section 4.4, and Miller (1991), the control strategy is essentially discontinuous.

\subsection{Stabilization}

Mărtensson (1985) has introduced an adaptive stabilizer for the following large class of linear systems where it is only assumed that there exists a dynamic stabilizing compensator of fixed order $l \in \mathbb{N}$.

Let $l \in \mathbb{N}$ and denote by $\mathcal{S}_{l}$ the set of all systems

$\dot{x}(t)=A x(t)+B u(t), y(t)=C x(t), x(0) \in \mathbb{R}^{n}$

$(A, B, C) \in \mathbb{R}^{n \times n} \times \mathbb{R}^{n \times m} \times \mathbb{R}^{p \times m}$ so that there exists

$(F, G, H, K) \in \mathbb{R}^{l \times l} \times \mathbb{R}^{l \times p} \times \mathbb{R}^{m \times l} \times \mathbb{R}^{m \times p}$

$\dot{z}(t)=F z(t)+G y(t), \quad u(t)=H z(t)+K y(t)$,

which asymptotically stabilizes $(A, B, C)$

Mártensson pointed out that the assumption on the existence of a dynamic feedback compensator is not crucial as is demonstrated in the following lemma.

LEMMA 5.1. Using the notations as in (5.1) we obtain that the linear dynamical system of order 1

$$
\dot{z}(t)=F z(t)+G y(t), \quad u(t)=H z(t)+K y(t), \quad z(0) \in \mathbb{R}^{l}
$$

is a dynamic feedback controller of the linear plant

$$
\dot{x}(t)=A x(t)+B u(t), \quad y(t)=C x(t), \quad x(0) \in \mathbb{R}^{n}
$$


if and only if the static feedback controller

$$
\tilde{u}(t)=\left[\begin{array}{cc}
K & H \\
G & F
\end{array}\right] \tilde{y}(t)
$$

is a controller of the system

$$
\dot{\tilde{x}}(t)=\tilde{A} \tilde{x}(t)+\tilde{B} \tilde{u}(t), \quad \tilde{y}(t)=\tilde{C} \tilde{x}(t), \quad \tilde{x}(0) \in \mathbb{R}^{n+l}
$$

where

$$
\begin{gathered}
\tilde{A}=\left[\begin{array}{cc}
A & 0 \\
0 & 0
\end{array}\right], \tilde{B}=\left[\begin{array}{cc}
B & 0 \\
0 & I
\end{array}\right], \tilde{C}=\left[\begin{array}{cc}
C & 0 \\
0 & I
\end{array}\right], \\
\tilde{x}=\left[\begin{array}{l}
x \\
z
\end{array}\right], \tilde{y}=\left[\begin{array}{l}
y \\
z
\end{array}\right], \tilde{u}=\left[\begin{array}{l}
u \\
\dot{z}
\end{array}\right]
\end{gathered}
$$

Note that (5.3) is stabilizable and detectable if, and only if, (5.5) is.

We do not present Mårtensson's (1985) original proof, that the knowledge of the order of a stabilizing controller is sufficient to design a universal adaptive stabilizer, the proof contains some gaps. The following version can be found in Logemann and Mårtensson (1990).

THEOREM 5.2. Let $l \in \mathbb{N}$ and $\sigma: \mathbb{R} \rightarrow \mathbb{N}$ be a piecewise constant right continuous function which satisfies

$$
\sigma([a, \infty))=\mathbb{N} \text { for all } a \in \mathbb{R}
$$

and which disconlinuity points are given by

$$
\tau_{i}=\tau_{i-1}^{2} \quad i=1,2, \ldots \quad, \tau_{0}>1 .
$$

Suppose $\mathcal{S} \subset \mathcal{S}_{l}$, sce (5.1), and $\mathcal{K}=\left\{K_{i}\right\}_{i \in \mathbb{N}} \subset \mathbb{R}^{(m+l) \times(p+l)}$ is a set of controllers associated with (5.4) so that for every $(A, B, C) \in \mathcal{S}$ there exists a $K_{i} \in \mathcal{K}$ which asymptotically stabilizes (5.5). Then the controller

$$
\tilde{u}(t)=K_{\sigma(k(t))} \tilde{y}(t), \quad \dot{k}(t)=\|\tilde{y}(t)\|^{2}+\|\tilde{u}(t)\|^{2}, \quad k(0) \in \mathbb{R}
$$

is a universal adaptive stabilizer for the class $\mathcal{S}$.

Proof. (Sketch) By (5.8) $k(t)$ is monotonically increasing. If $\sigma\left(k\left(t_{i}\right)\right)=\sigma\left(\tau_{i}\right)=$ $i_{0}$ hits a stabilizing feedback matrix $K_{i_{0}}$ then either the closed loop system stabilizes out on the time interval $\left[\tau_{i}, \tau_{i}^{2}\right)$ so that $\lim _{t \rightarrow \infty} k(t)<\tau_{i}^{2}$ and no more switching occurs, or it switches and goes unstable. But then condition (5.6) ensures that $\sigma(k)$ will hit the same $K_{i_{0}}$ again at a later switching point $\tau_{j}>\tau_{i}$. Because of $(5.7)$ the system will stay constant for a longer period. Eventually the system has enough time to settle down.

The result shows feasability rather than practical utility. If more information about the system class is available, then this information should be used to improve the transient behaviour and to simplify the regulation law. 
REMARK 5.3 .

(i) For a more general version of Theorem 5.2 see Section 6 .

(ii) It can be shown, see Lemma 4 in Logemann and Mårtensson (1990), that if $\mathcal{K}$ is a countable and dense subset of $\mathbb{R}^{(n+l) \times(p+l)}$ then, for every $(A, B, C) \in \mathcal{S}_{l}$, there exists a stabilizing dynamic compensator of the form (5.4) belonging to $\mathcal{K}$. Therefore $\mathcal{K}=\mathbb{Q}^{(n+l) \times(p+l)}$ satisfies the assumption of Theorem 5.2 .

(iii) If $\mathcal{K}$ in Theorem 5.2 is bounded, then the adaptation law in (5.8) can be simplified to $\dot{k}(t)=\|\tilde{y}(t)\|^{2}$.

Under the additional hypothesis that a compact subset of the class of systems (5.1) is considered, Fu and Barmish (1986) introduce an adaptive controller based on a piecewise constant switching strategy and providing Lyapunov stability and exponential decay of the solution.

An alternative control stategy to that presented in Theorem 5.2 resp. introduced by Mårtensson (1986) has been proposed by Miller and Davison (1987a, 1988), and for minimum phase systems by Miller and Davison (1991), see Section 4.3. The advantage of the different approach is that the adaptive controller tolerates certain disturbances and noise. Miller and Davison consider the following class of systems

$$
\left.\begin{array}{l}
\dot{x}(t)=A x(t)+B u(t), \quad y(t)=C x(t), \quad x(0) \in \mathbb{R}^{n} \\
(A, B, C) \in \mathbb{R}^{n \times n} \times \mathbb{R}^{n \times m} \times \mathbb{R}^{p \times n} \quad \text { is stabilizable and detectable }
\end{array}\right\}
$$

The idea is also based on an appropriate cycle through a parameter space and to use stepwise constant feedback. The following lemma gives an upper bound on the state $\tilde{x}(t)$ of the closed loop system in terms of $\tilde{y}(\cdot),\left.\tilde{u}(\cdot)\right|_{[0, t]}$ : for a proof see Miller and Davison (1987).

LEMma 5.4. Suppose the feedback law

$$
\tilde{u}(t)=\tilde{K} \tilde{y}(t) \quad, \tilde{K} \in \mathbb{R}^{(m+l) \times(m+p)}
$$

applied to (5.5) yields an exponentially stable closed loop system. Then there exist $M, \omega>0$, independent of $\tilde{x}(0)$ and $\tilde{u}(\cdot)$, such that the solution of $\dot{\tilde{x}}=[\tilde{A}+\tilde{B} \tilde{K} \tilde{C}] \tilde{x}$ satisfies

$$
\|\tilde{x}(t)\| \leq M\|\tilde{x}(0)\|+M \int_{0}^{t} e^{-\omega(t-s)}[\|\tilde{y}(s)\|+\|\tilde{u}(s)\|] d s \quad \text { for all } t \geq 0 .
$$

The inequality (5.11) is used to construct a criterion which decides whether to continue or to switch off the switching process. If the cycle through the parameter space hits a $K$ which exponentially stabilizes $(\tilde{A}, \tilde{B}, \tilde{C})$, then it follows from $(5.11)$ that for arbitrary $\beta>1$ and sufficiently large $i \in \mathbb{N}$

$$
\|\tilde{y}(t)\|<\left[\|\tilde{y}(0)\|+i+i k\left(t_{i}\right)\right] e^{-\beta^{-i}\left(t-t_{i}\right)} \quad \text { for all } t \geq t_{i}, t_{i} \geq 0
$$

where

$$
k(t):=\int_{0}^{t}\|\tilde{y}(s)\|+\|\tilde{u}(s)\| d s
$$


The inequality (5.12) is used as a main indicator for the switching procedure presented in the following proposition, see Miller and Davison (1987a, 1988).

THEOREM 5.5. Lel $l \in \mathbb{N}, \beta>1$ and

$$
h: \mathbb{N} \rightarrow \mathbb{R}^{(m+l) \times(m+p)}
$$

be a function so that $h(\mathbb{N})$ is dense in $\mathbb{R}^{(m+l) \times(m+p)}$. Then the regulation law

$$
\tilde{u}(t)=h(i) \tilde{y}(t), \quad \text { for } \quad t \in\left[t_{i}, t_{i+1}\right)
$$

where the adaptation mechanism is adjusted by the following sequence of switching times

$$
t_{i}:=\left\{\begin{array}{c}
\min \left\{t>t_{i-1}:\|\tilde{y}(t)\|=\left[i+\|\tilde{y}(0)\|+i k\left(t_{i-1}\right)\right] e^{-\beta^{-i}\left(t-t_{2-1}\right)}\right\} \\
\infty \quad \text { if the above minimum does not exist }
\end{array}\right.
$$

is a universal adaptive stabilizer for the class (5.9) under the additional assumption that it is known that there exists an $l^{\text {th }}$ order stabilizing compensator of the form (5.4).

Proof. (Sketch) Since im $h$ is dense in $\mathbb{R}^{(m+l) \times(m+p)}, h(i)$ will hit a stabilizing gain. If the decay rate $-\beta^{-i}$ in (5.12) is not slow enough, and/or the magnitude of $\|\tilde{y}(t)\|$ is too big, then a switching will occur. However, there is a time $t_{j}>t_{i}$ so that $h(j)$ is close to $h(i)$ with smaller decay rate and greater bound $j$, and finally (5.12) must be satisfied and no switching occurs any more. Then it can be proved that $\lim _{t \rightarrow \infty} x(t)=0$ and $r(\cdot)$ is bounded.

\section{REMARK 5.6 .}

(i) It is possible to modify the controller so that it becomes an adaptive stabilizer for the class of stabilizable and detectable systems, without the knowledge of the order of a stabilizing compensator. This can be achieved by an appropriate search of the controller dimension, of. Miller and Davison (1988).

(ii) If it is known that a correct $K_{i_{0}}$ lies in an open set $\mathcal{S} \subset \mathbb{R}^{(m+l) \times(m+p)}$, then $h$ can be replaced by some $\tilde{h}: \mathbb{N} \rightarrow \mathcal{S}$ so that $\tilde{h}(\mathbb{N})$ lies dense in $\mathcal{S}$.

(iii) The controller (5.14) can be modified so that it tolerates bounded disturbances $w_{1}(\cdot) \in \mathcal{C}_{p}$ in the output and bounded and Lebesque measurable $w_{2}(\cdot)$ in the input entering the system as in (1.1). The result is then that the signals $x(\cdot)$ and $r(\cdot)$ are bounded, only.

An important modification of the controller has been made by Miller and Davison (1989a). There the controller not only ensures exponential decay of the solution but also Lyapunov stability, that means for every $(A, B, C)$ belonging to (5.1) there exists $\omega, M>0$ not depending on the initial data so that the closed loop system satisfies (5.17). The controller does not have the shortcoming that the map $y \mapsto k$ is unbounded, cf. Section 3.5 , or requires a compactness assumption as in Fu and Barmish (1986). 


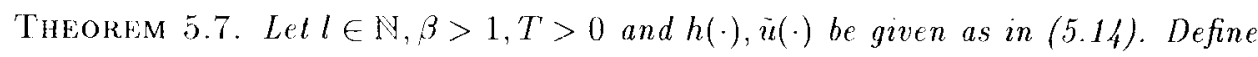

$$
t_{1}:=T, \quad \mu=e^{T} \int_{0}^{T}\|\tilde{y}(s)\|^{2} d s,\left.\quad \tilde{u}(\cdot)\right|_{[0, T]} \equiv 0 .
$$

Then the controller (5.14) with $t_{i}$ given by

$$
t_{i}:=\left\{\begin{array}{l}
\min \left\{t>t_{i-1}: \delta(t)=(i !) \mu e^{-\beta^{2} t}\right\} \\
\infty \text { if the above minimum does not exist }
\end{array}\right.
$$

where

$$
\delta(t)=\int_{t_{1}}^{t}\|\tilde{y}(s)\|^{2}+\|\tilde{u}(s)\|^{2} d s, \quad t>t_{1}
$$

is a universal stabilizer for the class (5.1). Moreover, for every $(A, B, C)$, there exists $M, \omega>0, i \in \mathbb{N}$, not depending on the initial conditions, such that

$$
\|\tilde{x}(t)\| \leq M e^{-\omega t}\|\tilde{x}(0)\| \text { for all } t \geq 0, \tilde{x}(0) \in \mathbb{R}^{n+m}
$$

and $h(i)$ switches at most $i$ times.

Proof. (Sketch) The proof is mainly based on the fact that if the closed loop system is exponentially stable, then $\delta(t)$ decays exponentially. $(i !) \mu e^{-\beta^{2} t}$ is a parametrized family of decaying exponentials increasing in absolute value and decreasing in decay rate as $i$ gets larger. Assume that the closed loop system is exponentially stable. If $i$ ! is not big enough or $\beta^{i}$ is not small enough then $\delta(t)$ may not lie below $(i !) \mu e^{-\beta^{2} t}$. In this case the switching procedure continues and comes back to a nearby feedback matrix, but now the bound $i$ ! is larger and the decay rate $-\beta^{i}$ is smaller. 'Thus eventually $\delta(t)$ stays below this exponential curve.

\subsection{Tracking and the servomechanism problem}

A first contribution to the adaptive tracking problem for non - minimum phase systems has been made by Mårtensson (1988). He pointed out that adaptive tracking of constant reference signals can be achieved for a given class of multivariable systems if a universal adaptive stabilizer is known and the class is invariant under precompensation by an integrator.

Miller and Davison (1991b) have shown how to implement an internal model so that an adaptive stabilizer results in an adaptive tracking controller. This approach can be extended to non minimum phase systems as well. For fixed $l \in \mathbb{N} \cup\{0\}$ consider the class

$$
\begin{aligned}
& \dot{x}(t)=A x(t)+B u(t)+E_{1} w, \quad y(t)=C x(t)+E_{2} w, \quad x(0) \in \mathbb{R}^{n} \\
& \text { for every } \\
& \left(A, B, C, E_{1}, E_{2}\right) \in \mathbb{R}^{n \times n} \times \mathbb{R}^{n \times m} \times \mathbb{R}^{p \times n} \times \mathbb{R}^{n \times n_{1}} \times \mathbb{R}^{p \times n_{1}}, m \geq p \\
& \text { there exists an } l^{t h} \text { order stabilizing compensator of the form (5.2) } \\
& \text { for the system }(3.30) \text {. }
\end{aligned}
$$


and the class of reference signals

$$
\mathcal{Y}_{\text {ref }}:=\left\{y_{\text {ref }} \in \mathcal{C}^{\infty}\left(\mathbb{R}, \mathbb{R}^{m}\right) \mid \quad \alpha\left(\frac{d}{d t}\right) y_{\text {ref }}(t) \equiv 0\right\}
$$

and of disturbance signals

$$
\mathcal{D}:=\left\{w \in \mathcal{C}^{\infty}\left(\mathbb{R}, \mathbb{R}^{n_{1}}\right) \mid \quad \alpha\left(\frac{d}{d t}\right) w(t) \equiv 0\right\}
$$

where the zeros of the monic $\alpha \in \mathbb{R}[s]$ are assumed to lie in $\overline{\mathbb{C}}_{+}$.

The following theorem by Miller and Davison (1991b) generalizes Martensson's result presented in Theorem 5.2 and 6.3 .

THEOREM 5.8. Let $l \in \mathbb{N} \cup\{0\}$, and suppose $m=p$. Then the adaptive controller (5.8) or (5.14) together with the compensator (3.29) is a universal adaptive servomechanism controller for the class of systems (5.18), of reference signals $\mathcal{Y}_{\text {ref }}$ and of disturbance signals $\mathcal{D}$.

Based on early results by Miller and Davison (1987a, 1988), an improved controller with smoother switching mechanism is presented in Miller and Davison (1991b). Here the internal model is not attached to the output as in Theorem 5.7, but instead it is attached to the error. It is only assumed that the numbers of outputs are equal or less than the number of inputs, but $\alpha(s)$ is restricted to have only simple zeros all of which lying on the imaginary axis. With $\hat{A}^{*}, \hat{B}^{*}$ given as in $(3.29)$ consider the compensator

$$
\dot{\xi}(t)=\hat{A}^{*} \xi(t)+\hat{B}^{*} e(t), \quad \xi(0) \in \mathbb{R}^{m p}, \quad e(t)=y_{r e f}(t)-y(t)
$$

Then the map $y_{r e f}:=\left[\begin{array}{c}y_{r e f} \\ w\end{array}\right] \mapsto y:=\left[\begin{array}{l}y \\ \xi\end{array}\right]$ is given by

$$
\dot{\bar{x}}(t)=\bar{A} \bar{x}(t)+\bar{B} u(t)+\bar{E} \ddot{y}_{r e f}(t), \quad \bar{y}(t)=\bar{C} \bar{x}(t)+\bar{F} \bar{y}_{r e f}(t), \quad x(0) \in \mathbb{R}^{m p+n}
$$

where

$$
\left.\begin{array}{lll}
\bar{A}=\left[\begin{array}{cc}
A & 0 \\
-\bar{B}^{*} C & \hat{A}^{*}
\end{array}\right], & \bar{B}=\left[\begin{array}{c}
B \\
0
\end{array}\right], & \bar{E}=\left[\begin{array}{cc}
E & 0 \\
-\hat{B}^{*} F & \hat{B}^{*}
\end{array}\right], \\
\bar{C}=\left[\begin{array}{cc}
C & 0 \\
0 & l
\end{array}\right], & \bar{F}=\left[\begin{array}{c}
F \\
0
\end{array}\right], & \bar{x}=\left[\begin{array}{l}
x \\
\xi
\end{array}\right]
\end{array}\right\}
$$

It can be shown that $(\bar{A}, \dot{B}, \bar{C})$ is stabilizable and detectable if $(A, B, C)$ is. Furthermore if a controller of the form (5.4) is applied to (5.21) or equivalently to the augmented system

$$
\dot{\tilde{x}}(t)=\tilde{A} \tilde{x}(t)+\tilde{B} \tilde{u}(t)+\tilde{E} y_{r e f}(t), \quad \tilde{y}(t)=\tilde{C} \tilde{x}(t)+\tilde{F} \ddot{y}_{r e f}(t), \quad \tilde{x}(0) \in \mathbb{R}^{n+l}
$$

where

$$
\begin{gathered}
\tilde{A}=\left[\begin{array}{cc}
A & 0 \\
0 & 0
\end{array}\right], \tilde{B}=\left[\begin{array}{cc}
\bar{B} & 0 \\
0 & 0
\end{array}\right], \tilde{C}=\left[\begin{array}{cc}
\bar{C} & 0 \\
0 & I
\end{array}\right] \\
\tilde{E}=\left[\begin{array}{c}
\bar{E} \\
0
\end{array}\right], \tilde{F}=\left[\begin{array}{c}
\bar{F} \\
0
\end{array}\right], \quad \tilde{K}=\left[\begin{array}{cc}
K & J \\
H & G
\end{array}\right]
\end{gathered}
$$


then the closed loop system is exponentially stable. So again it remains to find an algorithm such that $\tilde{K}$ cycles through $\mathbb{R}^{(m+l) \times(m+p)}$ and eventually stops if a 'correct' gain is hit.

THEOREM 5.9. Suppose $l \in \mathbb{N}$ and $\mathcal{K}=\left\{K_{i}\right\}_{i \in \mathbb{N}}$ is a dense subset of $\mathbb{R}^{(m+l) \times(m+p)}$. Then the controller

$$
\begin{aligned}
\dot{k}(t) & =\max \{-k(t)+\|\tilde{y}(t)\|+\|\tilde{u}(t)\|,\|e(t)\|\}, \quad k(0) \in \mathbb{R} \\
\dot{\xi}(t) & =\hat{A}^{*} \xi(t)+\hat{B}^{*} e(t), \quad \xi(0) \in \mathbb{R}^{m p} \\
\tilde{u}(t) & =h(k(t)) \tilde{y}(t) \\
h(k) & =\left\{\begin{array}{lll}
K_{1} & \text { if } \quad k<1 \\
K_{i} & \text { if } \quad k \in\left[i^{2},(i+1)^{2}\right), i \geq 2
\end{array}\right.
\end{aligned}
$$

is a universal adaptive regulator for all systems $\left(A, B, C, D, E_{1}, E_{2}\right)$ belonging to (5.18) and satisfying $r k\left[\begin{array}{cc}A-\lambda I & B \\ C & 0\end{array}\right] \geq n+p$ for all zeros $\lambda$ of $\alpha(\lambda)$, where $\alpha(\cdot)$ has simple zeros all of which lie on $i \mathbb{R}$, and for the class of reference signals $\mathcal{Y}_{\text {ref }}$ and disturbance signals $\mathcal{D}$.

The following different class of systems, note that $A$ is stable, has been considered by Miller and Davison $(1987,1989)$.

$$
\left.\begin{array}{l}
\dot{x}(t)=A x(t)+B u(t)+E_{1} w, y(t)=C x(t)+D u(t)+E_{2} w, x(0) \in \mathbb{R}^{n} \\
\left(A, B, C, D, E_{1}, E_{2}\right) \in \mathbb{R}^{n \times n} \times \mathbb{R}^{n \times m} \times \mathbb{R}^{p \times n} \times \mathbb{R}^{p \times m} \times \mathbb{R}^{n \times n_{1}} \times \mathbb{R}^{p \times n_{1}} \\
\sigma(A) \subset \mathbb{C}_{-}, \quad r k\left[D-C A^{-1} B\right]=p
\end{array}\right\}
$$

For this class, a so called 'low gain controller' is designed which solves the servomechanism problem for constant reference and disturbance signals. The switching strategy is similar to that used in Theorem 5.5. More importantly, Miller and Davison (1991c) have proved that the controller can be modified to achieve the same result under the additional constraint that the space $\bar{U}$ of input signals consists of bounded functions and that $y_{r e f}$ and $w$ satisfy a feasibility assumption in terms of $\left(A, B, C, D, E_{1}, E_{2}\right)$ and the bounds of $\bar{U}$.

\section{Necessary and sufficient conditions}

Byrnes et al. (1986) proved that if asymptotic stabilization can be achieved in an adaptive context, then Lyapunov stability can be achieved by a linear compensation if the system parameters are known. More precisely they proved the following necessary condition of universal adaptive stabilization.

THEOREM 6.1. If the feedback and adaptation rule

$$
u(t)=f(k(t), y(t)), \quad \dot{k}(t)=g(k(t), y(t)), \quad k(0) \in \mathbb{R}
$$

where $f: \mathbb{R}^{l} \times \mathbb{R}^{p} \rightarrow \mathbb{R}^{m}$ and $g: \mathbb{R}^{l} \times \mathbb{R}^{p} \rightarrow \mathbb{R}^{l}$ are $\mathcal{C}^{\infty}$ functions, is a universal adaptive stabilizer for a certain class $\Sigma$ of linear systems of the form

$$
\left.\begin{array}{l}
\dot{x}(t)=A x(t)+B u(t), \quad y(t)=C x(t), \quad x(0) \in \mathbb{R}^{n} \\
(A, B, C) \in \mathbb{R}^{n \times n} \times \mathbb{R}^{n \times m} \times \mathbb{R}^{t \times n}
\end{array}\right\}
$$


then the poles of each system belonging to $\mathrm{\Sigma}$ can be placed in $\overline{\mathbb{C}}_{-}$by some linear compensator of order $l$.

A sharpened version of this theorem which guarantees pole placement in $\mathbb{C}$ - is not available. Immediate consequences of Theorem 6.1 is the following corollary.

COROLlaRY 6.2. (i) There is no universal adaptive stabilizer of the form (6.1) of any order 1 for the class of stabilizable and detectable m-input, p-output linear systems.

(ii) There is no universal adaptive stabilizer of the form (6.1) for the class of singleinput single-output minimum phase systems.

The following result by Martensson (1985),(1986) shows that the order of any linear time-invariant stabilizing regulator is a sufficient information in order to carry out adaptive stabilization. This result is also an 'almost converse' of Theorem 6.1.

THEOREM 6.3. For every $l \in \mathbb{N}$ there exists a universal adaptive stabilizer of the form (6.1) and of order $l+1$ for the class of systems

$$
\begin{aligned}
& \dot{x}(t)=A x(t)+B u(t), y(t)=C x(t), x(0) \in \mathbb{R}^{n} \\
& (A, B, C) \in \mathbb{R}^{n \times n} \times \mathbb{R}^{n \times m} \times \mathbb{R}^{p \times m} \text { so that there exists } \\
& (F, G, H, K) \in \mathbb{R}^{l \times l} \times \mathbb{R}^{l \times p} \times \mathbb{R}^{m \times l} \times \mathbb{R}^{m \times p} \\
& \dot{z}(t)=F^{\prime} z(t)+G y(t), \quad u(t)=H z(t)+K y(t), \quad z(0) \in \mathbb{R}^{l} \\
& \text { which stabilizes }(A, B, C)
\end{aligned}
$$

Martensson (1985) also proved that Theorem 6.1 is no longer valid if the smoothness assumptions on $f$ and $g$ are dropped. By incorporating an appropriate search over the dimension of the controller into the adaptation mechanism, he obtains the following general result.

THEOREM 6.4. A universal adaptive controller exists which stabilizes all minimal systems of the form (6.2).

Recently Miller (1991) has shown that Theorem 6.1 is also no longer valid if timevarying stabilizers are allowed. He proved the existence of a smooth, time-varying, finite-dimensional, nonlinear, adaptive controller which stabilizes every linear, finitedimensional, stabilizable and detectable, time-invariant plant with a fixed number of inputs and outputs.

THEOREM 6.5. There exists an adaptive stabilizer of the form

$$
u(t)=f(k(t), y(t), t), \quad \dot{k}(t)=g(k(t), y(t), t), \quad k(0) \in \mathbb{R}
$$

where $f: \mathbb{R}^{l} \times \mathbb{R}^{p} \times \mathbb{R} \rightarrow \mathbb{R}^{m}, g: \mathbb{R}^{l} \times \mathbb{R}^{p} \times \mathbb{R} \rightarrow \mathbb{R}$ are infinitely differentiable functions, for the class of stabilizable and detectable systems of the form (6.2).

Proof. (Sketch) Based on the switching strategy introduced in Miller and Davison (1988), see (5.15), the controller is constructed in several steps. First a sampler is attached to the output and a zero-order-hold to the input of the stabilizable and detectable system $(A, B, C)$. For small enough sampling period the sampled 
discrete time system is stabilizable and detectable. Using a discrete time result, a linear periodic controller is given which exponentially stabilizes $(A, B, C)$. Now a countable dense subset of these controllers is constructed so that for each stablizable and detectable $(A, B, C)$ there exists a stabilizing linear periodic controller in this set. A switching algorithm between these linear time-varying controllers ensures the existence of an adaptive stabilizer for the whole class. Finally the discontinuous gain switching feedback is smoothed out.

Necessary conditions for the adaptive model reference control problem are given in Miller and Davison (1991a). There, the model reference problem coincides with the adaptive tracking problem for the class of reference signals which are produced by the output of a reference model exited by piecewise continuous bounded inputs. 'They consider the class of plants given by

$$
\left.\begin{array}{l}
\dot{x}(t)=A x(t)+b u(t), y(t)=c x(t), x(0) \in \mathbb{R}^{n} \\
(A, b, c) \in \mathbb{R}^{n \times n} \times \mathbb{R}^{n} \times \mathbb{R}^{1 \times n} \quad \text { is stabilizable and detectable }
\end{array}\right\}
$$

and the class of reference models $\left(A_{m}, b_{m}, c_{m}\right)$ which also belongs to $(6.4)$ and satisfy the additional assumption that $A_{m}$ is stable.

First, Miller and Davison (1991) show the following necessary and sufficient condition on the non-adaptive model reference control problem.

Proposition 6.6. The non adaptive model reference control problem is solvable under certain causality constraints if and only if

(i) the relative degree of $c(s I-A)^{-1} b$ is smaller or equal to the relative degree of $c_{m}\left(s I-A_{m}\right)^{-1} b_{m}$

(ii) every zero of $c(s I-A)^{-1} b$ in $\overline{\mathbb{C}}_{+}$is a zero of $c_{m}\left(s I-A_{m}\right)^{-1} b_{m}$ of the same or higher multiplicity.

In Rohrs et al. $(1982,1985)$ it is shown that many adaptive controllers will go unstable if the plant has some unmodelled high-frequency dynamics and a relative degree mismatch occurs. This is not surprising in the light of the following proposition, shown in Miller and Davison (1991a).

PROPOSITION 6.7. Necessary conditions for solvability of the adaptive model reference control problem for the class (6.4) are:

(i) A finite upper bound on the relative degree of all systems in (6.4) must be known.

(ii) The zeros of the uncertain plant on the imaginary axis must lie in a known finite set.

(iii) The model reference input is known or if not then the zeros of the uncertain plant in $\mathbb{C}_{+}$must lie in a known finite set. 


\section{Infinite dimensional systems}

The following results on universal controllers of infinite-dimensional linear systems generalize results which have been known before for finite-dimensional systems. Therefore these contributions can be viewed as robustness results showing that certain adaptive controllers which work for finite-dimensional systems are robust against certain infinite dimensional perturbations.

Most approaches use the well-known Willems-Byrnes controller

$$
u=N(k) k y, \quad \dot{k}=\|y\|^{2}
$$

where $N$ is a Nussbaum function, see $(2.10)$.

Logemann and Zwart (1991) consider the class of single-input single-output systems of the form

$$
\left.\begin{array}{l}
\dot{x}(t)=A x(t)+b u(t), \quad y(t)=c x(t), \quad x(0)=x_{0} \in X, \\
A \text { generates a strongly continuous semigroup } \\
\text { on a real Banach space } X, \\
b \text { and } c \text { are bounded linear operators and } c b \neq 0, \\
\text { the system has no zeros in } \operatorname{Re}(s) \geq \alpha \text { for some } \alpha<0, \\
(A, b) \text { is exponentially stabilizable, } \\
\operatorname{im} b \subset D(A), \quad \text { im } C^{*} \subset D\left(A^{*}\right),
\end{array}\right\}
$$

where $D(A)$ denotes the domain of $A$.

Under these assumptions they have shown that the Willems-Byrnes result carries over to infinite--dimensional systems.

THEOREM 7.1. Let $N: \mathbb{R} \rightarrow \mathbb{R}$ be a Nussbaum function, see (2.10). Then

$$
u(t)=N(k(t)) k(t) y(t), \quad \dot{k}(t)=y(t)^{2}, \quad k(0) \in \mathbb{R}
$$

is a universal adaptive stabilizer for the class (7.1).

Analogous results are proved for different system classes where the last assumption in (7.1) is replaced by one of the following conditions

$-\operatorname{im} b \subset D\left(A^{2}\right)$

$-\operatorname{im} c^{*} \subset D\left(A^{* 2}\right)$

- im $b \subset D(A)$ and $A$ generates an analytic semigroup

- im $c^{*} \subset D\left(A^{*}\right)$ and $A$ generates an analytic semigroup

Under much more restrictive assumptions ( $X$ a Hilbert space, $A$ selfadjoint and has a complete orthogonal system of eigenvectors and generates an analytic semigroup) on the class (7.1), Theorem 7.1 has been proved by Kobayashi (1987). However he considers multi-input multi-output systems but with the assumption that $\sigma(C B)$ is either lying in the right or left half plane.

Dahleh and Hopkins (1986) show that $u=N(k) k y, \dot{k}=y^{2}$ is a universal adaptive stabilizer for the class of single--input single-output 'minimum phase' delay systems satisfying a high frequency condition which generalizes the frequency domain relative degree 1 condition. This result has been extended to a considerable 
larger class of systems, feedback laws and gain adaptation mechanisms by Logemann and Owens (1988a), and to relative degree 2 systems by Dahleh (1989).

Byrnes (1987) considers infinite-dimensional linear systems with bounded infinitesimal generator $A$, thereby excluding 'all' interesting examples.

Dahleh (1988) considers the adaptive stabilization problem for a class of delay systems which is so restrictive, that interesting cases are not included. This has been pointed out by Logemann and Mårtensson (1991), Section 3.

Logemann and Owens (1988) developed an input-output theory of high gain adaptive stabilization of infinite-dimensional systems which covers in particular retarted systems and Volterra integrodifferential systems. A rich class of adaptation controllers which includes the Willems-Byrnes controller is applied to a large class of infinite-dimensional systems which satisfy a generalized minimum phase and relative degree one condition. Memoryless actuator and sensor nonlinearities are also considered in this approach, and a larger class of nonlinearities is allowed in Logemann (1990). Due to the large class of nonlinearities in the input and output lying either in a positive or negative sector, the usual Nussbaum function is restricted to the class of scaling-invariant Nussbaum functions. The results by Logemann and Owens (1988) include the results by Dahleh and Hopkins (1986), Kobayashi (1987), Byrnes (1989), Dahleh (1988). In Logemann (1990) an adaptive stabilizer for retarded systems is introduced which ensures exponential decay of the solution of the closed loop system at the price that the minimum phase condition has to be strengthened, see Section 3.5.

Logemann and Mårtensson (1990) consider the so called Pritchard Salamon class of infinite-dimensional systems with unbounded control and observation and assume that the systems are exponentially stabilizable and exponentially detectable. By using switching function controllers the results by Martensson (1985), (1986), see Section 5.1, are extended to the infinite-dimensional case. The paper is not based on a high-gain concept, however an application to minimum phase systems is given.

A contribution to the adaptive servomechanism problem for infinite-dimensional systems has been made by Logemann and Ilchmann (1991). A class of multi-input multi-output systems in an input-output description is considered including retarded and integrodifferential systems which contain the class of finite-dimensional minimum phase systems with high frequency gain $\operatorname{det}(C B) \neq 0$. The paper extends the finite-dimensional stabilization results by Mårtensson (1986) and Byrnes and Willems (1984) to infinite-dimensional systems. Moreover the universal adaptive regulator solves the servomechanism problem for a class of reference signals consisting of solutions of a differential equation and asymptotic rejection of disturbance signals including $L_{2}$-functions. The universal regulator uses the internal model principle as discribed in Section 3.6. 


\section{Nonlinear systems}

Mårtensson (1990) considers the universal adaptive stabilization problem for the class of single--input single-output first order systems of the form

$$
\left.\begin{array}{l}
\dot{x}(t)=f(x(t))+g(x(t)) u(t), \quad x(0) \in \mathbb{R} \\
f, g \in \mathcal{C}^{\infty}(\mathbb{R}), \quad f(0)=0, \quad g(x) \neq 0 \quad \text { for all } \quad x \in \mathbb{R}
\end{array}\right\}
$$

A necessary condition for adaptive stabilization is given and two universal adaptive stabilizers are presented. The adaptation and feedback law is chosen piecewise $\mathcal{C}^{\infty}$, instead of a piecewise constant control produced by an algorithmic procedure as presented in Theorem 5.2.

Adaptive stabilizers for the class (8.1) are also given by Nikitin and Schmid (1990). In addition they show exponential decay of the solution.

In Khalil and Saberi (1987) and Saberi and Lin (1990) a class of multi-input multi-output nonlinear systems of known strong relative degree $p \geq 1$ is considered. This is a generalization of linear minimum phase systems of relative degree $p$ where the spectrum of the high frequency matrix is known to lie in $\mathbb{C}_{+}$. The class covers in particular $p$ th-order vector differential equations of the form

$$
y(t)^{(p)}=f\left(y(t), \ldots, y(t)^{(p-1)}\right)+M\left(y(t), \ldots, y(t)^{(p-2)}\right) u(t)
$$

where $M$ is uniformly bounded and positive definite and $f$ is Lipschitzian. The adaptive stabilizer consists of a stabilizable and observable realization of a compensator so that the closed loop system is of relative degree 1 , cf. Section 4.1, and a gain adaptation which produces a piecewise constant gain $k(\cdot)$ which is increasing as long as the output does not satisfy a certain convergence criterion. The idea is similar to that used for many adaptive stabilizers of linear systems: try a constant high-gain feedback, if the gain is not high enough a convergence criterion decides to switch to a higher gain, eventually the closed loop system is stable so that the convergence criterion is satisfied and no more switching occurs.

In several contributions, E.P. Ryan considers large classes of nonlinear system and introduces controllers which are based on the Willems-Byrnes controller together with a Nussbaum function. In Ryan (1990) the following class of nonlinear systems is considered.

$$
\begin{aligned}
& \dot{x}(t)=A x(t)+f(x(t))+a z(t), \quad \dot{z}(t)=g(x(t), z(t))+b u(t) \\
& (x(0), z(0)) \in \mathbb{R}^{n} \times \mathbb{R}, a \in \mathbb{R}^{n}, f \in \mathcal{C}\left(\mathbb{R}^{n}, i m a\right) \text { is linearly bounded } \\
& \langle c, a\rangle b \neq 0 \text { for a known } c,(A, b, c) \in \mathbb{R}^{n \times n} \times \mathbb{R}^{n} \times \mathbb{R}^{1 \times n} \text { is minimum phase } \\
& g \in \mathcal{C}\left(\mathbb{R}^{n} \times \mathbb{R}, \mathbb{R}\right) \text { satisfies }|g(x, z)| \leq \beta \gamma(x, z) \text { for all }(x, z) \in \mathbb{R}^{n} \times \mathbb{R}, \\
& \text { for an unknown } \beta>0 \text { and known } \gamma \in \mathcal{C}\left(\mathbb{R}^{n} \times \mathbb{R}, \mathbb{R}_{+}\right)
\end{aligned}
$$

Note that, by assumption, the nonlinear system is a perturbation of a linear minimum phase system of relative degree 1.

As opposed to all previously discussed feedback strategies, Ryan (1990) introduces a universal adaptive feedback stabilizer using discontinuous feedback of $x(t), z(t)$, essentially a sign function, interpreted as a set-valued map. Thus the 
general framework uses the theory of differential inclusions. The stability results are obtained by using Lyapunov functions and an extension of LaSalle's invariance principle. A similar approach using discontinuous feedback but for a less general class than (8.2) has already been introduced by Ryan (1988).

In the same spirit, Ryan (1991) introduces a universal adaptive stabilizer for the rich class of nonlinear systems modelled by a scalar $n$-th order differential inclusion of the form

$$
\left.\begin{array}{l}
\alpha z^{(n)}(t)+\beta u(t) \in \mathcal{G}\left(z(t), \ldots, z^{(n-1)}(t)\right), \quad\left(z(0), \ldots, z^{(n-1)}(0)\right) \in \mathbb{R}^{n} \\
y \mapsto \mathcal{G}(y) \text { is continuous on } \mathbb{R}^{n} \text { and takes convex and compact values in } \mathbb{R}, \\
\alpha \beta \neq 0 \text { and the full state }\left(z(t), \ldots, z^{(n-1)}(t)\right) \text { is available for feedback }
\end{array}\right\}
$$

This is an extension of results presented in Ryan (1991a). An adaptive stabilizer for an extension of the class (8.3) and the following class (8.4) to multi-input, multi-output systems is presented in Ryan (1991b).

The power of the discontinuous feedback approach is demonstrated in Ryan (1992) where he considers the class of nonlinearly-perturbed, multi-input, multioutput systems of the form

$$
\left.\begin{array}{l}
\dot{x}(t)=A x(t)+B[f(t, x(t))+u(t)]+g(t, x(t)), \\
y(t)=C x(t), \quad x(0) \in \mathbb{R}^{n} \\
(A, B, C) \in \mathbb{R}^{n \times n} \times \mathbb{R}^{n \times m} \times \mathbb{R}^{m \times n} \text { is minimum phase, } \\
\sigma(C B) \subset \mathbb{C}_{-} \text {or } \subset \mathbb{C}_{+} \\
f, g \text { are Carathéodory } \\
\text { and satisfy, for a known continuous } \phi \text { and unknown } \mu>0 \\
\|f(t, x)\| \leq \mu \phi(C x) \text { and }\|g(t, x)\| \leq \gamma \text { for all } x \text { and almost all } t .
\end{array}\right\}
$$

It is shown that the simple adaptive controller (suitably interpreted)

$$
\left.\begin{array}{l}
u(t)=N(k(t))\left[e(t)+\left(1+\phi(y(t))\|e(t)\|^{-1} e(t)\right], \quad e(t)=y(t)-y_{\text {ref }}(t)\right. \\
\dot{k}(t)=\|e(t)\|^{2}+(1+\phi(y(t))\|e(t)\|
\end{array}\right\}
$$

where $N(\cdot)$ is a Nussbaum function, is a universal adaptive tracking controller for the class $(8.4)$ and for each reference signal $y_{r e f}(t)$ which is absolutely continuous on compact intervals and has essentially bounded derivative. Surprisingly this approach is not based on the internal model principle and the class of reference signals is fairly rich.

\section{Conclusion}

Over the last 15 years much progress has been made in the field of non-identifierbased adaptive control. Necessary conditions have been given for the existence of adaptive controllers, and various adaptive controllers have been designed for different classes of mainly finite dimensional linear systems and also some nonlinear and infinite dimensional systems. The nonlinear structure of the controllers has become simpler.

We have also seen that, roughly speaking, the adaptive controllers can be divided into two classes, one which uses smooth feedback and adaptation laws, the other 
where the gain in the feedback law is tuned in a piecewise constant manner. The first approach is more 'elegant' in a mathematical way, the latter one is quite often easier to analyse. 'There is no obvious reason to give preference to one approach over the other.

Adaptive control for finite dimensional linear systems is quite well understood, many universal adaptive controllers having been presented for different classes of systems (multi-input multi-output, minimum phase as well as non-minimum phase, known or unknown relative degree) that not only solve the stabilization problem but also solve the tracking and servomechanism problems. Robustness properties have been addressed in most cases.

The extension of these results to infinite dimensional systems is mainly restricted to the stabilization problem, necessary conditions for the adaptive stabilization problem are not available. Adaptive control for nonlinear systems is still in its beginnings. Most authors consider nonlinearly perturbed linear minimum phase systems.

One shortcoming of many universal adaptive controllers is that they show only feasibility, with performance of the nonlinear closed loop system being unpredictable and dependent, in an erratic way, on the initial data. The design of controllers so that the transient behaviour is improved has only started, and the prediction of the transient behaviour in terms of the initial conditions is an open problem. For example, simulations have shown that the terminal system (3.21) is in most cases exponcntially stable. Is there a generic property hidden behind this observation?

There is also a poor understanding how the design of the controller can take into account inputs and outputs corrupted with noise. More importantly, what can be achieved, if the class of input signals is constrained?

Finally, a comparison between non -identifier and identifier based adaptive control necds further investigation.

\section{Acknowledgements}

The author is indebted to H. Logemann of the University of Bremen and to D.E. Miller of the University of Waterloo for careful reviews of this article and for providing detailed lists of misprints, errors, and comments. Many thanks also go to D.H. Owens and S. Townley of the University of Exeter and to E.P. Ryan of the University of Bath for useful suggestions on various aspects.

'The work was supported by the FEC SCIENCE programme under grant number SC-1-0433-C-(A), by the Eniversity of Exeter, and mainly by the German Research Association (I)FG).

\section{REFERFNCHS}

As'rö̈m, K.J. 1987 Adaptive feedback control. Procedings of the $1 E E E, 75,18.5-$ 217

BYrnes, C.I. 1987 Modelling and algorithmic issues in intelligent control. In Ewing, R.E. et al, editors, The Merging of Descriptions, New York, Springer Verlag. Byrnes, C.I., Helmke, IT. \& MORsk, A.S. 1986 Necessary conditions in adaptive control. In Byrnes, ( $:$ I. \& Linquist, A., editors, Modelling Identification and Robust Control, North-Holland, pages 3-14.

Brrnes, (.$I$ \& IsIDori, A. 1986 Asymptotic expansions, root-loci and the global 
stability of nonlinear feedback systems. In Fliess, M. \& Hazewinkel, M., editors, Algcbraic and Geometric Methods in Nonlinear Control Theory, D.Reidel Publishing Company, pages 159-179.

Byrnes, C.I. \& Willems, J.C. 1984 Adaptive stabilization of multivariable linear systems. Proc. 23rd Conf. on Decision and Control, Las Vegas, pages 1574-1577.

Cabrera, J.B.D. \& Furuta, K. 1989 Improving the robustness of Nussbaumtype regulators by the use of $\sigma$-modification - local results, Systems 8 Control Letters, 12, 421-429.

Corless, M. 1988 First order adaptive controllers for systems which are stabilizable via high gain output feedback. In Byrnes, C.I., Martin, C.F. \& Saeks, R.E., editors, Analysis and Control of Nonlinear Systems, North-Holland, pages 13-16.

Corless, M. 1991 Simple adaptive controllers for systems which are stabilizable via high gain output feedback. IMA Journal of Math. Control \& Information, this issue.

DAHLEH, M. 1988 Sufficient information for the adaptive stabilization of delay systems. Systems 8 Control Letters, 11, 357-363.

DAHLEH, M. 1989 Generalizations of Tychonov's theorem with applications to adaptive control of SISO delay systems. Systems 8 Control Letters, 13, 421-427.

Dahleh, M. \& Hopkins, W.E. JR. 1986 Adaptive stabilization of single-input single-output delay systems. IEEE Transactions on Automatic Control, AC-31, $577-579$.

Feuer, A. \& Morse, A.S. 1978 Adaptive control of single-input, single-output linear systems. IEEE Transactions on Automatic Control, AC-23, 557-569.

FU, M. \& BARMISH, B.R. 1986 Adaptive stabilization of linear systems via switching control. IEEE Transactions on Automatic Control, 31, 1097-1103.

HELMKE, U. \& PRÄTZEL-Wolters, D. 1988 Stability and robustness properties of universal adaptive controllers for first order linear systems. Int. J. Control, 48, 1153-1182.

Helmke, U. \& Prätzel-Wolters, D. 1988a Stability and robustness properties of universal adaptive controllers for first order linear systems. In Byrnes, C.I., Martin, C.F. \& Saeks, R.E., editors, Analysis and Control of Nonlinear Systems, North-Holland, pages 17-26.

Helmke, U., Prätzel-Wolters, D. \& Schmid, S. 1990 Adaptive tracking for scalar minimum phase systems. In Hinrichsen, D. \& Mårtensson, B., editors, Control of Uncertain Systems, Boston, Birkhäuser, pages 101-118.

Heymann, M., Lewis, J.H. \& Meyer, G. 1985 Remarks on the adaptive control of linear plants with unknown high-frequency gain. Systems $\mathcal{G}$ Control Letters, $\mathbf{5}$, $357-362$.

ILCHMANN, A. \& LogemanN, H. 1991 High-gain adaptive stabilization of multivariable linear systems - revisited. Report 242, Institut für Dynamische Systeme, Universität Bremen, to appear in Systems $\&$ Control Letters.

ILCHMAnN, A. \& OWEns, D.H. 1990 Adaptive stabilization with exponential decay. Systems 8 Control Letters, 14, 437-443.

IlchmanN, A. \& OWENS, D.H. 1991 Exponential stabilization using non-differential gain adaptation. IMA Journal of Math. Control and Information, 7, 339-349. 
LCCHMAN, A. \& OWENs, D.H. 1991 a Robust universal adaptive stabilization in the presence of nonlinearities. Pror. 1st European Control Conference, Grenoble, pages $2580-2585$.

ILCHMANN, A., OWEns, D.H. \& Prä́tZel, Wolters, D. 1987 High gain robust adaptive controllers for multivariable systems. Systems 8 Control Letters. 8, $397-$ 404.

IOANNou, P. 1986 Adaptive stabilization of not necessarily minimum phase plants. Systems \& Conlrol Letters, 7, 281-287.

KHALIL, H.K. \& SABERI, A. 1987 Adaptive stabilization of a class of nonlinear systems using high gajn feedback. IEEE Transactions on Automatic Control, AC$32,1031-1035$.

KоBAYASHI. 'T. 1987 Global adaptive stabilization of infinite-dimensional systems. Systems \& Control letters, $9,215-223$.

LogkmanN, H. 1990 Adaptive exponential stabilization for a class of nonlinear retarded processes. Mathematics of Control, Signals, and Systems, 3, 255-269.

Logemann, H. \& ILCHMANN, A. 1991 An adaptive servomechanism for a class of infinite dimensional systems. Report 243, Institut für Dynamische Systeme, Universität Bremen.

LogEMANn, H. \& MÄrTEnsson, B. 1990 Adaptive stabilization of infinite dimensional systems. Report 230, Institut für Dynamische Systeme, Universität Bremen. Logemann, H. \& OWEns, D.H. 1988 Input-output theory of high-gain adaptive stabilization of infinite dimensional systems with non linearities. Int. J. Adaptive Control and Signal Processing, 2, 193-216.

LOGEMANN, H. \& OWENS, D.II. 1988a Robust and adaptive high-gain control of infinite-dimensional systems. In Byrnes, C.I., Martin, C.F. \& Saeks, R.E., editors. Analysis and Control of Nonlinear Systems, North-Holland, pages 35-44

LogEmanN, H. \& ZWART, H. 1991 Some remarks on adaptive stabilization of infinite dimensional systems. Systems \& Control Letters, 16, 199-207.

MAREELS, I. 1984 A simple self-tuning controller for stably invertible systems. Systems 8 Control Letters, 4, 5-16.

MARTENSSON, B. 1985 The order of any stabilizing regulator is sufficient a priori information for adaptive stabilization. Systems $\&$ Control Letters, 6, 87-91.

Martensson, B. 1986 Adaptive Stabilization. PhD Thesis, Lund Institute of Technology, Lund, Sweden.

Martensson, B. 1987 Adaptive stabilization of multivariable linear systems. Contemporary Mathematics, 68, 191-225.

MARTEnsSon, B. 1988 Adaptive stabilization without high-gain. In Lecture Notes in Control 6 Info. Sciences, 105, New York, Springer-Verlag, pages 226-238 (also in Proc. IIASA Conf., 1986).

MARtensson, B. 1990 Remarks on adaptive stabilization of first order non-linear systems. Systems \& Control Letters, 14, 1-7.

Miller, D.E. 1991 Adaptive stabilization using a nonlinear time-varying controller. preprint.

Miller, D.F. and Davison, E.J. 1987 The self-tuning robust servomechanism problem. Proc. 26th Conf. on Decision and Control, Los Angeles, pages 384-390.

Miller, D.E. \&. DAvison, E.J. 1987a A new self-tuning controller to solve the 
servomechanism problem. Pros. Bthth Conf, on Dreision and Control, Los Angcles, pages $843-849$

Milier, I).H. \& Davison, E..J. 1988 An adaptive controller which can stabilize any stabilizable and detectable LII system. In Byrnes, C.I., Martin, (..F. \& Saeks, R.E., editors, Analysis and Control of Nonlinear Systems, North-Holland, pages $51-58$.

MILAK, D.F. \& DAVISON. F..J. 1989 The self tuning robust servomechanism problen. IEEE Transactions on Automatic Control, AC-34, 511-52:3.

MHLer, 1).E. \& IJAISON, E.J. 1989a An adaptive controller which provides Lyapunov stability. IELE Transactions on Automatic Control, AC-34, 599-609.

MILLE, D.F. \& DAvison, E.J. 1991 An adaptive controller which provides an arbitrarily good transient and steady-state response. IEFE Transactions on Automatic Control, AC-36, 68-81.

Mulfer, I).F. \& I MVISON, E.J. 1991 a On necessary assumptions in continuous time model reference adaptive control. Preprint (a shortened version is in Proc. Psth Conf. on Derision and Control, Tampa, Florida, pages 1573-1578, 1989).

MiLLFR, D.F. \& DAVison, E.J. 1991b An adaptive tracking problem. Preprint. Milak, D.E. \& DAVISON, F.J. 1991 c An adaptive tracking problem with a control input constraint. Preprint.

Morst, A.S. 1980 Global stability of parameter adaptive control systems. HEE Transartions, AC-25, 433-439.

MoRsk, A.S. 198:3 Recent problems in parameter adaptive control. In Landan, I.I). editor, Outils et Modiles Mathematiques pour l'Automatique, l'Analyse de Systrme t te Traitment du Signal, Vol. 3, Editions du CNRS, Paris, pages 733-740; also in Proc. CNRS Colloquium on Development and Vtilization of Math. Models in Automat. Control, Bell-Iste, France, 1982.

MoRsE, A.S. 1984 An adaptive control for globally stabilizing lincar systems with unknown high frequency gains. Lect. Notes in Control Inf. Scicnces, 62, pages $58-68$, Berlin, Springer Verlag.

Morse, A.S. 1981 a New dircctions in parameter adaptive control. Proc. 29rd Conf. on Decision and Control, Las Vegas, pages 1566-1568.

MORSE, A.S. 1985 A three-dimensional universal controller for the adaptive stabilization of any strictly proper minimum-phase system with relative degree not exceding two. IEEE Transactions on Automatic Control, AC-30,1188-1191.

MORSE, A.S. 1987 A 4( $n+1)$-dimensional model reference adaptive stabilizer for any relative degree one or two, minimum phase system of dimension $n$ or less. $A u$ tomatica, 23, 123-125.

Morse, A.S. 1987a High-gain feedback algorithms for adaptive stabilization. Proc of the 5th Yale Workshop on Applications of Adaptive Systems Theory, pages
13-18.

Morse, A.S. 1988 Simple algorithms for adaptive stabilization. Lect. Notes in Control 8 Info. Sciences, 105, pages 254-264, Berlin, Springer-Verlag; also in Proc. IIASA Conf. on Modelling and Adaptive Control, Sopron, Hungary, 1986.

MORSE, A.S. 1990 Towards a unified theory of parameter control: tunability. IEEL Transactions, AC-35, 1002-1012.

Morse, A.S. 1990a Towards a unified theory of parameter control-Part 2: cer- 
tainty equivalence and implicit tuning. Report 9012, Dept. of Flectrical Engineering, Yale University.

Mudiet'T, D.R. \& Morse, A.S. 1985 Adaptive stabilization of linear systems with unknown high frequency gains. IEEE Transactions on Automatic Control, AC-30, 549-554.

Mudiet', D.R. \& Morse, A.S. 1989 A smooth, high gain adaptive stabilizer for linear systerns with known relative degree. Proc. American Control Conference, Pillsburgh. PA, pages 21-26.

Narfindra, K.S., Lin, Y.H. \& Valavani, L.S. 1980 Stable adaptive controller design, Part II: Proof of stability. IEEE Transactions on Automatic Control, AC$25,440-448$.

Nikitin, S. \& Schmid, S. 1990 Universal adaptive stabilizers for one-dimensional nonlinear systems. Report 44, Fachbereich Mathematik, Universität Kaiserslautern. Nussbatm, R.D. 1983 Some remarks on a conjecture in parameter adaptive control. Systems 6 Control Letlers, 3, 243-246.

Owexs, D.H. 1991 Adaptive stabilization of a class of singular systems. 2nd Inter. Symposium on Robust and Implicit Systems, Technical University of Warsaw.

Owhins, D.H., Prätzel-Wolters, D. \& Ilchmann, A. 1987 Positive-real structure and high-gain adaptive stabilization. IMA J. of Math. Control G Information. 4, $167-181$.

Prätzel-Wolters, D., OWens, D.H. \& llchmann, A. 1989 Robust stabilization by high gain feedback and switching. Int. J. Control, 49, 1861-1868.

Rohrs, F., Valvani, L., Athans, M. \& Stein, G. 1982 Robustness of adaptive control algorithms in the presence of unmodeled dynamics. Proc. 21st IEEE Conf. on Decision E Control, Orlando, pages 3-11.

Rohrs, F., Valvani, I., Athans, M. \& Stein, G. 1985

Robustness of continuous-time adaptive control algorithms in the presence of unmodeled dynamics, IEEE Transactions on Automatic Control, AC-30, 881-889.

RYAN, E.P. 1988 Adaptive stabilization of a class of uncertain nonlinear systems: A differential inclusion approach. Systems $\&$ Control Letters, 10, 9;)-101.

RYAX, F.P. 1990 Discontinuous feedback and universal adaptive stabilization. In Hinrichsen, D. \& Martensson, B., editors, Control of Uncertain Systems, Boston, Birkhäuser, pages 245-258.

RYAX, L.P. 1991 A universal adaptive stabilizer for a class of nonlinear systems. Systems \& Control Letters, 16, 209-218.

Rran, E.P. 1991a Finite lime stabilization of uncertain nonlinear planar systems. Dymamic's and Control. 1. 83-94.

RyAN, E.P. 1991b Adaptive stabilization of multi input nonlinear systems.

Preprint.

Ryan, E.P. 1992 Universal $W^{1, \infty}$-tracking for a class of nonlincar systems. To appear in Systems 8 Control Letters.

SABERI, A.\& LIN, Z. 1990 Adaptive high-gain stabilization of 'minimum phase' nonlinear systems. Proc. 29th IEEE Conf.on Decision 8 Control, Honolulu, Hawait, pages $3417-3422$.

Scrmu, S. 1991 Adaptive Synchronization of Interconnected Systems. PhD Thesis, Fachbereich Mathematik, Universität Kaiserslautern. 
SontaG, E.D. 1990 Mathematical Control Theory, Deterministic Finite Dimensional Systems. New York, Springer-Verlag.

TaO, G. \& Ionnnou, A. 1991 Robust adaptive control of plants with unknown order and high frequency gain. Int. J. Control, 53, 559-578.

ToWnley, S. \& OWEns, D.H. 1991 A note on the problem of multi-variable adaptive tracking. IMA J. of Math. Control \& Information, this issue.

Willems, J.C. \& Byrnes, C.I. 1984 Global adaptive stabilization in the absence of information on the sign of the high frequency gain. Lect. Notes in Control $\mathcal{B}$ Info. Sciences, vol. 62, pages 49-57, Berlin, Springer-Verlag.

Wonham, W.M. 1979 Linear Multivariable Control: a Geometric Approach. New York, Springer--Verlag.

ZHU, X.-J. 1989 A finite spectrum unmixing set for $G L_{3}(\mathbb{R})$. In Bowers, K. \& Lund, J., editors, Computation and Control, pages 403-410, Boston, Birkhäuser. 